\title{
THERMORESPONSIVE, REDOX-POLYMERIZED CELLULOSIC HYDROGELS UNDERGO IN SITU GELATION AND RESTORE INTERVERTEBRAL DISC BIOMECHANICS POST DISCECTOMY
}

\author{
D.M. Varma ${ }^{1}$, H.A. Lin ${ }^{1}$, R.G. Long ${ }^{2}$, G.T. Gold ${ }^{1}$, A.C. Hecht ${ }^{2}$, J.C. Iatridis ${ }^{2}$ and S.B. Nicoll ${ }^{1, *}$ \\ ${ }^{1}$ Department of Biomedical Engineering, The City College of New York, New York, NY, USA \\ ${ }^{2}$ Leni and Peter W. May Department of Orthopedics, Icahn School of Medicine at Mount Sinai, \\ New York, NY, USA
}

\begin{abstract}
Back and neck pain are commonly associated with intervertebral disc (IVD) degeneration. Structural augmentation of diseased nucleus pulposus (NP) tissue with biomaterials could restore degeneration-related IVD height loss and degraded biomechanical behaviors; however, effective NP replacement biomaterials are not commercially available. This study developed a novel, crosslinked, dual-polymer network (DPN) hydrogel comprised of methacrylated carboxymethylcellulose (CMC) and methylcellulose (MC), and used in vitro, in situ and in vivo testing to assess its efficacy as an injectable, in situ gelling, biocompatible material that matches native NP properties and restores IVD biomechanical behaviors. Thermogelling MC was required to enable consistent and timely gelation of CMC in situ within whole IVDs. The CMC-MC hydrogel was tuned to match compressive and swelling NP tissue properties. When injected into whole IVDs after discectomy injury, CMC-MC restored IVD height and compressive biomechanical behaviors, including range of motion and neutral zone stiffness, to intact levels. Subcutaneous implantation of the hydrogels in rats further demonstrated good biocompatibility of CMC-MC with a relatively thin fibrous capsule, similar to comparable biomaterials. In conclusion, CMC-MC is an injectable, tunable and biocompatible hydrogel with strong potential to be used as an NP replacement biomaterial since it can gel in situ, match NP properties, and restore IVD height and biomechanical function. Future investigations will evaluate herniation risk under severe loading conditions and assess long-term in vivo performance.
\end{abstract}

Keywords: Intervertebral disc, nucleus pulposus, cellulosic hydrogel, injectable, discectomy.

*Address for correspondence: Steven B. Nicoll, Ph.D., Department of Biomedical Engineering, The City College of New York, Steinman Hall, Room 401, 160 Convent Avenue, New York, NY 10031, USA.

Telephone: +1 2126506237 Email: snicoll@ccny.cuny.edu

Copyright policy: This article is distributed in accordance with Creative Commons Attribution Licence (http://creativecommons.org/licenses/by-sa/4.0/).

\section{Introduction}

Intervertebral disc (IVD) degeneration is strongly associated with back pain, affecting $15-30 \%$ of the US population at estimated annual costs of $\$ 194$ billion (Becker et al., 2010; Jacobs, 2008). The IVD consists of the centrally located nucleus pulposus (NP) and peripherally located annulus fibrosus (AF). The NP is a highly hydrated tissue that functions to bear compressive loads and maintain the IVD height by means of swelling pressures. The AF resists spinal motions and NP swelling by bearing tensile stresses through its fibrous and laminated structure. With IVD degeneration there is a loss of constituent matrix molecules, such as proteoglycans and collagen, leading to non-uniform load distributions in the IVD that manifest as cracks and fissures in the AF and can result in herniation (Adams et al., 1996). IVD herniation is a common condition that is characterized by extrusion of the NP through the AF and is highly associated with clinically painful conditions (Endean et al., 2011; Weber, 1994). Discectomy is a procedure widely performed to treat IVD herniations and involves the removal of the extruded NP tissue to prevent its contact with surrounding nerves. It is clinically favorable to non-operative controls in providing pain relief but does not restore IVD biomechanical behaviors that are altered by the loss in IVD height and intradiscal pressure, which result from NP herniation and AF damage. There is also a recurrence rate of 5-15\% (Kim et al., 2009; McGirt et al., 2009; Shin, 2014). Advancement of IVD degeneration following damage, herniation and/or discectomy may require patients to undergo spinal fusion, which also has deleterious biomechanical consequences on motion segments adjacent to the 
treated segments (Kim et al., 2009). Furthermore, recent explant studies indicate that degenerated IVDs that undergo discectomy are more susceptible to hypermobility in the motion segment, in turn increasing spinal instability (Showalter et al., 2014). All of these conditions highlight a need for biomaterialbased strategies that are capable of repairing and restoring function of injured and degenerated IVDs.

Replacing degraded or herniated NP tissue with a biomaterial could limit progression of degeneration and reduce painful conditions. NP replacement strategies have evolved with early generations employing preformed devices that required invasive procedures for implantation. Many devices failed clinically due to device migration and extrusion from the herniation site (Lewis, 2012; Di Martino et al., 2005). AF sealants and closure devices can address device migration concerns (Guterl et al., 2013; Parker et al., 2016). However, AF repair strategies alone may not fully restore IVD biomechanics. Recently developed NP replacements include hydrogels, because of their high water-content, tunable material properties, and ability to conform to the intradiscal space (Balkovec et al., 2013; Gan et al., 2017; Pérez-San Vicente et al., 2017; Showalter et al., 2015).

Design objectives for an ideal hydrogel-based, NP replacement biomaterial include: injectability with rapid in situ gelation, appropriate for a minimally invasive clinical setting; stable formation of gels that mimic healthy NP material properties, restoring IVD height and multiple biomechanical behaviors of injured IVDs; and biocompatibility (Iatridis et al., 2013). Although injectable hydrogels have been developed, few studies have assessed the ability of such gels to restore disc mechanical function. Cannella et al. demonstrate restoration of select biomechanical properties with physically crosslinked polyvinyl alcohol (PVA)-polyvinylpyrrolidone hydrogels (Cannella et al., 2014). Generally, PVA hydrogels are pre-crosslinked by means of physical crosslinks formed using repetitive freeze-thaw cycles (Cannella et al., 2014; Joshi et al., 2006) or chemically, using crosslinkers such as diglycidyl ether (Binetti et al., 2014). They have limited capability to conform to the disc space post injury or nucleotomy, in comparison to in situ gelling materials. As a result, these hydrogels may be more susceptible to herniation. An in situ gelling tripolymeric hydrogel developed by Smith et al. exhibits robust mechanical properties, and restores compressive biomechanics in a fatigue model and a preclinical model (Gullbrand et al., 2017; Malhotra et al., 2012; Showalter et al., 2015; Smith et al., 2014). However, this Schiff basedependent hydrogel requires $>10 \mathrm{~h}$ of crosslinking time to achieve peak biomechanical properties, which may limit its clinical translation, given that rapid gelation is a key requirement.

Carboxymethylcellulose (CMC)-based hydrogels can meet many of the design criteria for NP replacements. CMC is a water-soluble derivative of cellulose with an extensive safety and biocompatibility profile, as it has been FDA-approved for use in the pharmaceutical and food industries (Heinze and Koschella, 2005). CMC mimics the NP, with an exaggerated water absorbing capacity at physiological $\mathrm{pH}$, due to the carboxyl groups on the polymer backbone. Unlike other commonly used biopolymers (such as collagen and hyaluronic acid), CMC does not undergo enzymatic degradation in humans, providing enhanced stability. Moreover, modification of the hydroxyl groups along the CMC backbone with methacrylate moieties allows covalent coupling of the polymer chains in the presence of radical initiators, forming stable crosslinked hydrogels (Reza and Nicoll, 2010). In vitro characterization of photocrosslinked methacrylated CMC demonstrates that CMC hydrogels have functional properties comparable to native NP and can support stem cell differentiation towards an NP phenotype (Gupta et al., 2011; Gupta and Nicoll, 2013; Gupta and Nicoll, 2014; Lin et al., 2016). However, UV-based crosslinking methods have limited clinical applicability for IVD repair due to poor deep tissue penetration and potential harm to the surrounding tissue.

The development and characterization of an injectable CMC-based hydrogel for NP repair is an important step required to advance towards clinical translation. A fully injectable CMC hydrogel was previously developed by covalently crosslinking with water-soluble redox initiators, ammonium persulfate (APS) and tetramethylethylene diamine (TEMED). This redox-initiated CMC hydrogel matches NP properties and forms stable hydrogels in vitro (Varma, 2016). However, redox-polymerized $\mathrm{CMC}$ failed to gel when injected into large IVDs in situ post discectomy, due to extravasation of the hydrogel solution from the IVD upon injection. To overcome this challenge to clinical translation, a novel dual-polymer network (DPN) consisting of modified $\mathrm{CMC}$ and methacrylated methylcellulose (MC) was developed. The inherent thermogelation property of $\mathrm{MC}$ at $>32{ }^{\circ} \mathrm{C}$ increased the viscosity of the redox-initiated DPN solution at body temperature, enabling consistent and reliable gelation in situ in the microenvironment of large IVDs (Fig. 1). As such, the objectives of this study were to: 1) develop a novel injectable, thermogelling, redox-polymerized CMC-MC hydrogel with material properties akin to the native NP; 2) determine if the CMC-MC hydrogel could restore native biomechanics of the bovine IVD post discectomy; and 3) assess CMC-MC hydrogel cytocompatibility using $3 \mathrm{D}$ cell culture and biocompatibility in a rat subcutaneous pouch model.

\section{Materials and Methods}

\section{Polymer synthesis}

High and low molecular weights of CMC (SigmaAldrich) at $250 \mathrm{kDa}$ and $90 \mathrm{kDa}$, were used in combination with an MC (Sigma-Aldrich) polymer 

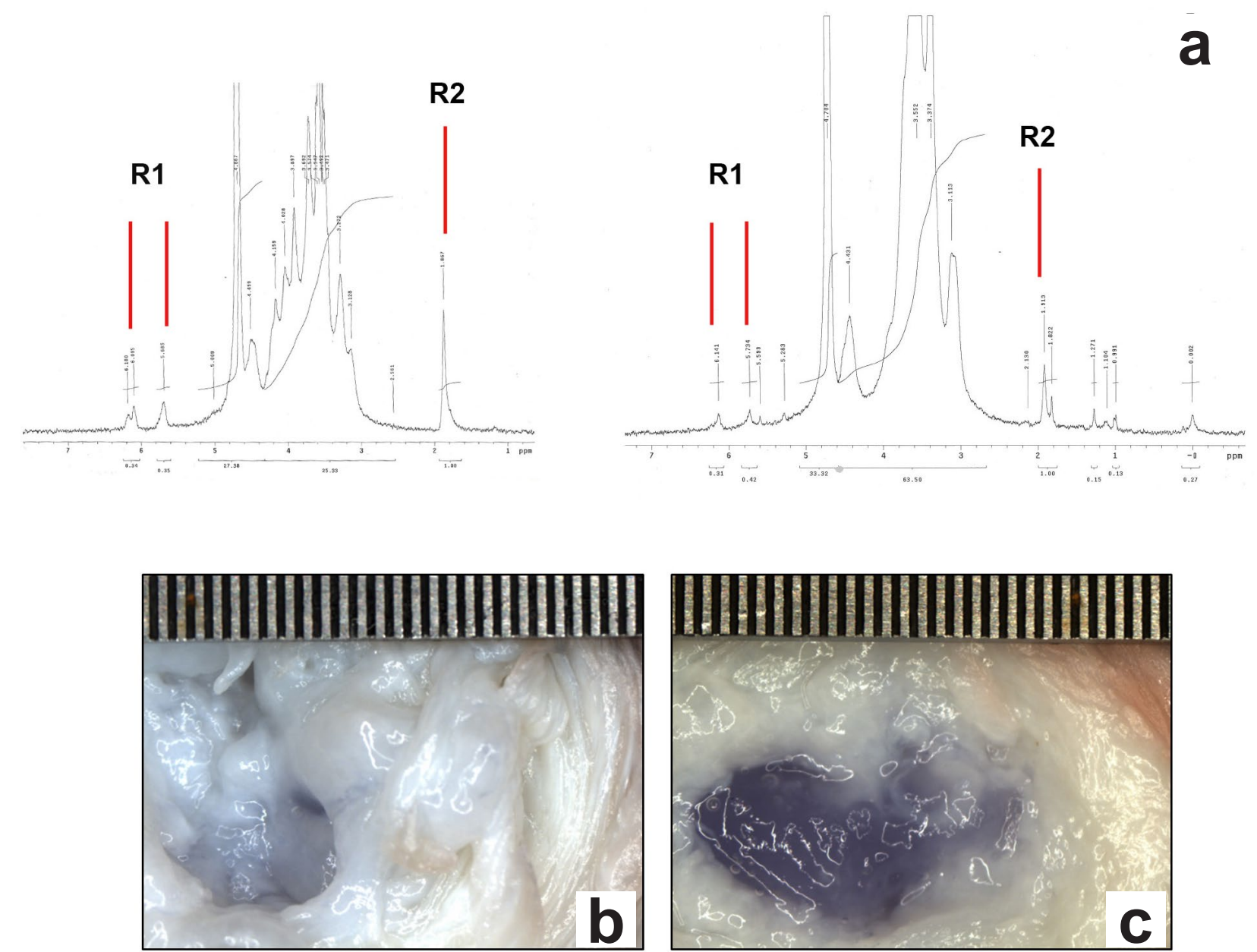

Fig. 1. In situ gelation within nucleotomized IVDs occurred with redox-initiated CMC-MC crosslinked DPN but not with CMC alone. (a) Representative ${ }^{1} \mathrm{H}-\mathrm{NMR}$ spectra of CMC (left) and MC (right) modified with methacrylate groups on the polymer backbone. R1 and R2 indicate the methylene and methyl protons on the methacrylate group, respectively. (b) No in situ gelation was observed using redox-initiated $\mathrm{CMC}$, as exemplified by a $3 \%(w / v) 90 \mathrm{kDa}$ CMC solution alone with $20 \mathrm{~mm}$ APS/TEMED initiators, where a slight blue-coloring occurred in the native NP region of the IVD which had been disrupted from discectomy. (c) In situ gelation always occurred with CMC-MC DPN, as shown by a $3 \%(\mathrm{w} / \mathrm{v}) 90 \mathrm{kDa}$ CMC- $3 \%(\mathrm{w} / \mathrm{v})$ $15 \mathrm{kDa}: 41 \mathrm{kDa}(1: 1) \mathrm{MC}$ solution with $20 \mathrm{~mm}$ APS/TEMED initiators, wherein a blue gel had filled the NP region after discectomy. Trypan blue dye was used for visualization since CMC and MC are clear. Scale in mm.

blend of two molecular weights, $15 \mathrm{kDa}$ and $41 \mathrm{kDa}$, in a $1: 1$ ratio for this study. The low-high molecular weight blend of $\mathrm{MC}$ was selected to provide adequate control over solution viscosity and handling. Methacrylation of CMC and MC polymers was performed by the esterification of hydroxyl groups with methacrylic anhydride (Sigma-Aldrich) at a pH of 8.5, as described by Reza and Nicoll (2010). The CMC and MC reaction products were purified by dialysis and the degree of methacrylation was quantified using ${ }^{1} \mathrm{H}-\mathrm{NMR}(300 \mathrm{MHz}$, Varian Mercury 300) following acid hydrolysis of both modified polymers (Gold et al., 2014; Varma et al., 2014). Briefly, $20 \mathrm{mg}$ of the modified polymer dissolved in $20 \mathrm{~mL}$ of deionized water was hydrolyzed at a $\mathrm{pH}$ of 2 for 2-3 $\mathrm{h}$ at $80^{\circ} \mathrm{C}$. After cooling, the $\mathrm{pH}$ of the solutions was adjusted to 7 and the hydrolyzed polymer was extracted after lyophilization. The dried product was dissolved in $1 \mathrm{~mL}$ of deuterium dioxide solvent and analyzed for methacrylate peaks using ${ }^{1} \mathrm{H}-\mathrm{NMR}$. Molar percentage of methacrylation was determined by the relative integrations of the methacrylate proton peaks (methylene peak, $\delta=6.2$ and 5.8 ppm; methyl peak, $\delta=2.0 \mathrm{ppm}$ ) with respect to the carbohydrate backbone.

\section{Part I: In situ gelation and hydrogel characterization}

Characterization of the CMC-MC hydrogel occurred following in situ gelation within IVD motion segments to be consistent with the intended minimally invasive clinical application of the biomaterial. Bovine caudal IVDs were used since they are readily available, commonly accepted in the literature, contain a fibrous NP and exhibit several properties similar to human IVDs (Alini et al., 2008). Discectomy was performed on healthy bovine IVDs to approximate current clinical practice as well as to produce a 
void into which the hydrogel could be injected. The resulting biomechanical changes have been found to mimic IVDs undergoing degeneration (Showalter et al., 2014). CMC-MC DPNs were then injected in situ into the IVDs, before being isolated and prepared to uniform geometry for in vitro hydrogel characterization tests.

Bovine explant sample preparation and discectomy injury Bone-disc-bone motion segments (cc2-3 and cc3-4) were harvested from healthy, skeletally mature bovine tails obtained from a local abattoir. The segments were prepared by sawing parallel cuts through the vertebral bodies (Likhitpanichkul et al., 2014). A cruciate incision was made posterolaterally with a \#15 blade through the AF. The NP was dislodged with a curette and approximately 0.15$0.20 \mathrm{~g}(\approx 80 \%)$ of NP tissue was removed with a pituitary rongeur via the $2 \mathrm{~mm}$ incision (Malhotra et al., 2012).

\section{In situ hydrogel fabrication}

CMC-MC formulations of varying macromer concentrations, molecular weights and redox initiator concentrations, as listed in Table 1, were screened for their ability to gel in situ within injured bovine motion segments post injection through a $20 \mathrm{G}$ needle coupled with a dual-barrel syringe and mixing tip. A customcasting device was used to fabricate hydrogels in vitro for comparison to in situ cured gels.

Solutions of methacrylated CMC and MC in Dulbecco's Phosphate Buffered Saline (DPBS) (Thermo Fisher Scientific, USA) were combined and prepared in dual-barrel syringes (Pearson Dental Supply Co., Sylmar, CA, USA). The redox initiators, APS and TEMED, were added to separate barrels of the syringe such that they mixed in the mixing tip upon injection. Trypan blue was added to detect the presence of the hydrogel in the motion segment post gelation. The uncrosslinked mixture was warmed to $37^{\circ} \mathrm{C}$ in a water bath and injected with a $20 \mathrm{G}$ needle (Becton, Dickinson \& Co., Franklin Lakes, NJ, USA) into the NP void of the injured motion segments via the injury site. The implanted motion segments were incubated in a water bath at $37^{\circ} \mathrm{C}$ for $30 \mathrm{~min}$.

\section{Hydrogel isolation and material characterization}

Following in situ gelation, motion segments were dissected along the endplate and the hydrogel was carefully extracted and assessed for the material properties. Upon extracting the hydrogel from the motion segment, the sample was carefully separated from the NP and the outer portion of the hydrogel was excised to exclude any of the adhering NP tissue. Cylindrical hydrogel specimens ( $3 \mathrm{~mm}$ diameter, $\approx 2 \mathrm{~mm}$ thickness) were cored from CMC-MC hydrogels using a 3-mm biopsy punch and a custommade cutting guide made with blades $2 \mathrm{~mm}$ apart.

\section{Mechanical testing}

Hydrogels ( $n=7 ; 1$ gel each from 7 independent in situ gelation experiments in bovine motion segments) underwent mechanical loading in unconfined compression in a DPBS bath using an established protocol (Reza and Nicoll, 2010), consisting of a creep test ( $1 \mathrm{~g}$ tare load at $10 \mu \mathrm{m} / \mathrm{s}$ ) followed by a multi-ramp stress relaxation (three $5 \%$ strain ramps with relaxation intervals of 2,000 s). The average equilibrium Young's modulus $\left(E_{y}\right)$ was determined as the slope of the equilibrium stress versus strain curves at $5 \%, 10 \%$ and $15 \%$ strain. The equilibrium stress $\left(\sigma_{e q}\right)$ and peak stress $\left(\sigma_{p k}\right)$ were calculated at the $15 \%$ strain ramp and were used to calculate the \% relaxation.

$$
\% \text { relaxation }=1-\frac{\sigma_{e q}}{\sigma_{p k}}
$$

The same protocol was used to measure the $E_{y}$ of $\mathrm{CMC}_{90} \mathrm{MC}$ hydrogels formed within a casting device.

\section{Swelling ratio}

The wet weight $\left(W_{s}\right)$ of the cored hydrogels was measured after an overnight incubation in DPBS at $37^{\circ} \mathrm{C}$. The gels were lyophilized to obtain the dry weights $\left(W_{d}\right)(n=7)$. The swelling ratio, $\left(Q_{w}\right)$, was obtained as follows (Reza and Nicoll, 2010):

$$
Q_{w}=\frac{W_{s}}{W_{d}}
$$

\section{Rheology}

Rheological analysis was performed on the base polymers, methacrylated $\mathrm{CMC}_{90}$ and $\mathrm{CMC}_{250^{\prime}}$ at $37^{\circ} \mathrm{C}$ to determine the impact of the polymer molecular weight on the injectability and mixing of their corresponding DPN with methacrylated MC. An AR2000ex (TA Instruments) rheometer equipped

Table 1. Formulations evaluated for in situ gelation. A range of polymer solutions was prepared by varying the molecular weight and concentration of macromers (CMC and MC), and the redox initiator (APS and TEMED) concentration.

\begin{tabular}{|c|c|c|c|c|}
\hline \multicolumn{2}{|c|}{$\begin{array}{c}\text { Molecular weight } \\
\text { (kDa) }\end{array}$} & \multicolumn{2}{|c|}{$\begin{array}{c}\text { Macromer concentration } \\
\text { (w/v) }\end{array}$} & $\begin{array}{c}\text { Redox initiator } \\
\text { concentration (mM) }\end{array}$ \\
\hline CMC & MC & CMC & MC & APS/TEMED \\
\hline 90 & $15: 41$ & $2 \%, 3 \%$ & $3 \%$ & $15,20,25$ \\
\hline 250 & $15: 41$ & $2 \%, 3 \%$ & $3 \%$ & $15,20,25$ \\
\hline
\end{tabular}


with a cone and plate geometry $\left(2^{\circ}, 20 \mathrm{~mm}\right)$ was used to record measurements. Optimal test parameters ( $1 \%$ strain at $1 \mathrm{~Hz}$ frequency) were selected by means of strain and frequency sweep measurements on the base polymer solution $(3 \%(\mathrm{w} / \mathrm{v}))$ in DPBS. The test parameters were obtained from the linear viscoelastic region where $G^{\prime}$ and $G^{\prime \prime}$ are independent of frequency and strain (Winter and Chambon, 1986). The complex viscosity values were obtained from a time sweep conducted over $300 \mathrm{~s}$.

A similar protocol was used to record the gelation kinetics and complex viscosity of the CMC-MC DPN, where gelation completion time was defined as the first time four consecutive points exhibited less than a $2 \%$ change in $\mathrm{G}^{\prime}$.

\section{Statistical analysis}

A one-way ANOVA with a Tukey's post-hoc test was used to determine the effect of CMC molecular weight on mechanical properties and $Q_{w}$ of the CMC-MC hydrogels and the viscosity of the base polymers $(p<0.05)$. Data represent the mean \pm standard deviation (SD).

Part II: Biomechanical restoration post discectomy The $\mathrm{CMC}_{90} \mathrm{MC}$ formulation together with APS and TEMED at $20 \mathrm{~mm}$ had the most favorable characteristics for an NP replacement, and thus, was selected for further biomechanical evaluation.

\section{Study design}

Fourteen motion segments were randomly divided into two groups: explants receiving the $\mathrm{CMC}_{90} \mathrm{MC}$ hydrogel implant following injury were labeled 'Experimental' and designated as 'Implanted' in the reported results, and the control samples receiving no hydrogel implant after injury were referred to as 'Sham' and termed 'No Implant'. Each motion segment underwent a mechanical loading regimen under three conditions, Intact, Injured (discectomy, as described in Part I) and Implanted/No Implant, with overnight incubation in DPBS and protease inhibitors at $4{ }^{\circ} \mathrm{C}$. This repeated measures design (Fig. 2a, b,c) was used to eliminate variations between animals and IVD levels (Malhotra et al., 2012). The $\mathrm{CMC}_{90} \mathrm{MC}$ hydrogel solution $(500-750 \mu \mathrm{L})$ was injected into the void space of the injured IVDs in the Experimental group via a $20 \mathrm{G}$ needle, and the motion segments (Experimental and Sham groups) were incubated at $37{ }^{\circ} \mathrm{C}$ for $30 \mathrm{~min}$. The solution was injected until it exuded out of the injury site to ensure filling of the NP with the hydrogel. A radiopaque dye (Isovue ${ }^{\circledR}$, Bracco, Monroe Township, NJ, USA) was mixed with the hydrogel solutions to visualize implant location post gelation.

\section{Specimen preparation and mechanical testing}

Seven bovine tails were used for the study with two motion segments per tail (cc2-3, cc3-4) assigned to the Experimental or Sham group. All of the musculature and soft tissue were removed, along with the facet and transverse processes, to exclude their contribution to IVD mechanics. The initial and post-test height, and average diameters of the motion segments were determined by $x$-ray imaging and caliper measurements, respectively, after which motion segments were potted in poly(methyl methacrylate) (Likhitpanichkul et al., 2014).

Mechanical testing was performed at room temperature on an MTS servohydraulic system (Bionix 858, MTS, Eden Prairie, MN, USA), equipped with a DPBS bath. Samples were tested under load control and underwent an initial 10 s $30 \mathrm{~N}$ preload followed by 25 sinusoidal cycles at $0.1 \mathrm{~Hz}$ between - $-0.5 \mathrm{MPa}$ compression and $0.25 \mathrm{MPa}$ tension followed by a slow ramp compression from 0 to $170 \mathrm{~N}$ at 1 N/s (Johannessen et al., 2004; Malhotra et al., 2012) (Fig. 2d). The peak compressive stress of $0.5 \mathrm{MPa}$ was chosen based on the physiological spinal load measured in the human lumbar spine in an unsupported standing position (Wilke et al., 1999), while the peak tensile stress of $0.25 \mathrm{MPa}$ was sufficient to measure the NZ and tensile properties of the IVD (Likhitpanichkul et al., 2014). Slow ramp compression was incorporated into the testing protocol to measure the isolated mechanical loading response of the NP, as it typically bears low loads in the IVD at low frequencies (1 N/s) (Johannessen et al., 2006).

\section{Biomechanical analyses}

The $25^{\text {th }}$ cycle of the tension-compression loading regimen was used for data analysis to ensure dynamic equilibrium was attained (Johannessen et al., 2006). The axial range of motion ( $\mathrm{ROM}$ ) was calculated as the total peak-to-peak displacement. A custom MATLAB (Mathworks, Natick, MA) program was used to fit the load-displacement data to a sixth order polynomial to measure the NZ parameters. The point of minimum slope on this fit was located and the NZ stiffness was calculated as the slope of the loading data at this point (Showalter et al., 2014). The compressive and tensile stiffness were calculated from a linear regression of the load-displacement curve between $60-100 \%$ of the loading curve maximum load and $80-100 \%$ of the unloading curve maximum load, respectively (Malhotra et al., 2012). The NZ length was measured as the displacement between the intersections of the compressive and tensile fits to the NZ fit. Linear regression of the slow ramp load-displacement curve was used to obtain the slow ramp compressive stiffness (Johannessen et al., 2004; Johannessen et al., 2006) (Fig. 2e,f). It is noteworthy that the mechanical parameters calculated from tests at $0.1 \mathrm{~Hz}$ have some viscoelastic contributions and should be considered 'dynamic' and not 'equilibrium' moduli parameters.

The IVD height was calculated by using a cubic spline function in a custom MATLAB code to measure the average distance between the top and bottom traced endplates on the X-Ray radiographs (Likhitpanichkul et al., 2014). 

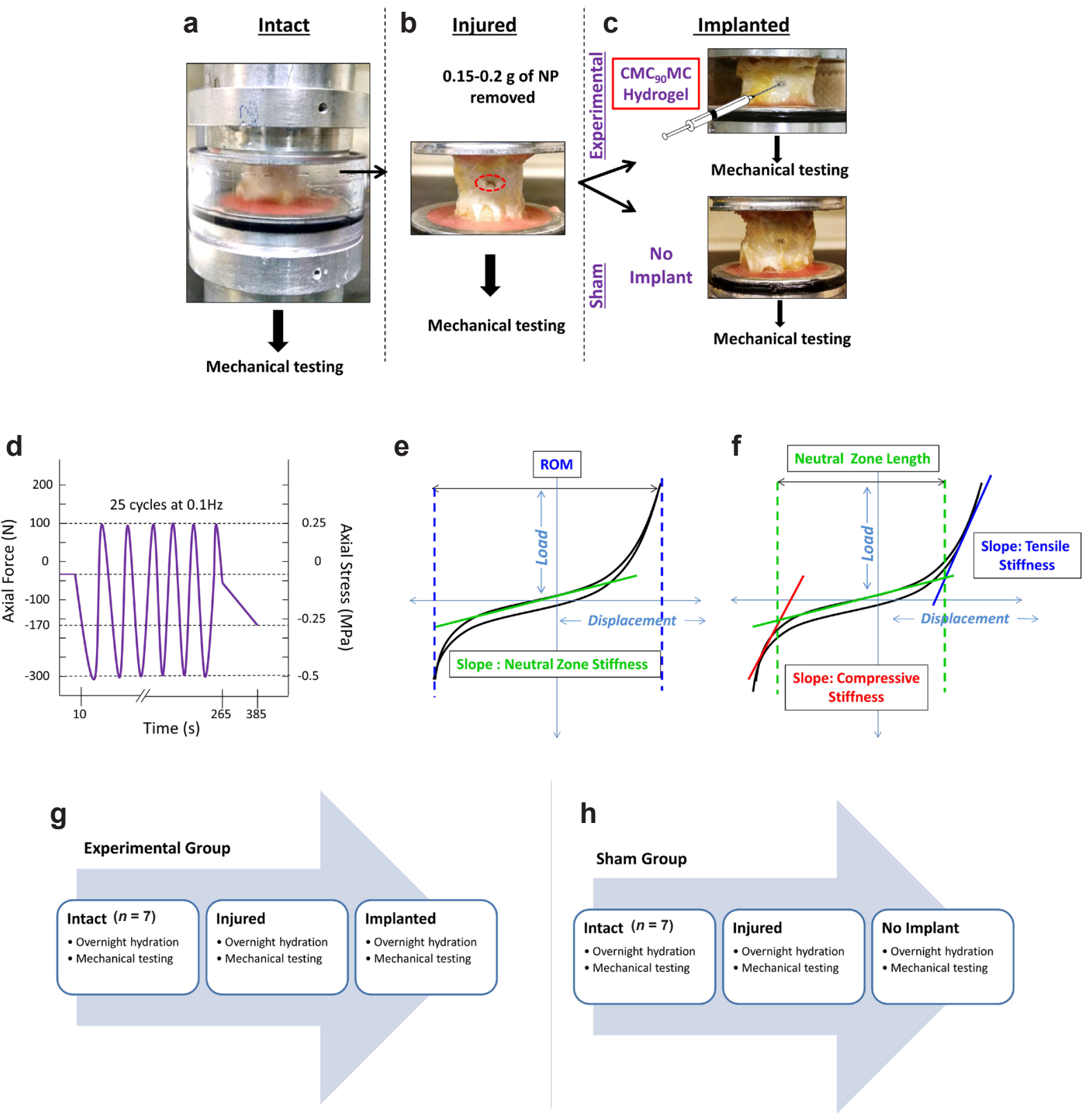

Fig. 2. Study design and biomechanical testing protocol for bovine motion segments. The motion segments were tested using a repeated measures design including three conditions. (a) Intact (b) Injured (post discectomy) (c) Implanted ( $\mathrm{CMC}_{90} \mathrm{MC}$ hydrogel implant was injected in the Experimental group while the Sham group received no implant). Representative schematics describing the (d) mechanical testing protocol used to test the explants at Intact, Injured and Implanted conditions. (e,f) Biomechanical parameters measured post mechanical testing. ( $g$ and $\mathbf{h}$ ) Study regimen used for the Experimental and Sham groups over a period of $3 \mathrm{~d}$.

\section{Histology}

After the third round of mechanical testing, the samples from the Experimental and Sham groups were fixed in a buffered zinc formalin solution (Z-Fix, Anatech, Battle Creek, MI, USA). The IVDs were sawed off of the vertebral bodies and dehydrated using 2-propanol (Thermo Fisher Scientific), cleared with methyl salicylate (Sigma-Aldrich), infiltrated and embedded in methyl methacrylate, and polymerized over 3-4 d. Sections of 4-6 $\mu \mathrm{m}$ thickness were cut using a sledge microtome (Leica SM2500, Leica Biosystems, Buffalo Grove, IL, USA) and mounted on charged slides. Sections were deplasticized by two rounds of incubation in xylene for $30 \mathrm{~min}$ followed by a $1: 1$ mixture of Xylene and ethylene glycol monoethyl ether (EGME, Thermo Fisher Scientific) for $5 \mathrm{~min}$. After washing the sections with fresh EGME followed by tap water rinsing, the sections were stained with toluidine blue (Sigma-Aldrich) for $15 \mathrm{~min}$, rinsed with tap water, and cover slips were placed on stained sections with mounting medium (Laudier et al., 2006).

\section{Statistical analysis}

Data are presented as the mean \pm SD. Repeated measures ANOVA with a Tukey's post-hoc test were performed to compare biomechanical properties 
between all paired combinations (i.e., Intact $v s$. Injured, Intact vs. Hydrogel Implant/No Implant, Injured vs. Hydrogel Implant/No Implant) $(p<0.05$ considered significant).

\section{Part III: Biological response}

\section{Cytocompatibility}

Human dermal fibroblasts were seeded in $\mathrm{CMC}_{90} \mathrm{MC}$ hydrogels, polymerized with $20 \mathrm{mM}$ APS/TEMED at a density of $12 \times 10^{6}$ cells/mL and cultured for $6 \mathrm{~d}$. Dermal fibroblasts were used as a general screen for connective tissue cytocompatibility, given the potential for CMC-MC gels to be employed for other clinical indications (i.e., soft tissue reconstruction), as well as for comparison to our prior studies evaluating related cellulosic gels (Gold et al., 2014; Gold et al., 2015). Direct-contact cytotoxicity testing by cell encapsulation was utilized since our previous experiments reveal poor cell adhesion and migration of NP cells, marrow-derived mesenchymal stromal cells and dermal fibroblasts seeded on CMC (Gupta et al., 2011; Reza and Nicoll, 2010). This is likely attributed to the highly negatively-charged, hydrophilic environment of the CMC polymer, which results in poor serum protein adsorption due to strong water molecule binding to the material (Vogler, 2012). Similar models are employed by other research groups to assess cytocompatibility of biomaterials (Mironi-Harpaz et al., 2012; Shin et al., 2012). CMC (3\% w/v) hydrogels, fabricated using a $10 \mathrm{mM}$ redox initiator concentration at the same cellseeding density were used as controls, based on our earlier studies which show good cytocompatibility using this formulation (Varma, 2016). All hydrogels $(n=5)$ were cultured at $37{ }^{\circ} \mathrm{C}$ and $5 \% \mathrm{CO}_{2}$ in high glucose DMEM with $1 \%$ Pen/Strep (Gibco, United States) and $10 \%$ FBS (Gibco, United States). Total DNA content was measured to assess cell proliferation using the PicoGreen assay (Molecular Probes, Eugene, OR) on $\mathrm{d} 1$ and 6. The samples were lyophilized, homogenized and digested in pepsin, prepared in $0.05 \mathrm{~N}$ acetic acid for $48 \mathrm{~h}$ at $4{ }^{\circ} \mathrm{C}$ (SigmaAldrich). The digested solution was then neutralized with 10× Tris-buffered saline. The samples were read on a BioTek Instruments plate reader (Synergy 4, Winooski, VT, USA) at an excitation/emission of 480/520 nm. Calf thymus DNA (Sigma-Aldrich) was used to create a standard curve (Gupta et al., 2011). Additionally, the cell viability in the hydrogels was assessed on days 1 and 6 using Live/Dead assay (Invitrogen, Thermo Fisher Scientific, USA) staining with calcein AM and ethidium homodimer-1.

\section{Biocompatibility}

The foreign body response to the $\mathrm{CMC}_{90} \mathrm{MC}$ hydrogels, created with varying concentrations of APS and TEMED $(0,10$ and $20 \mathrm{mM})$, was assessed using a subcutaneous pouch model in male Sprague Dawley rats $(n=4)$ (Charles River, Kingston, NY, USA) weighing 250-300 $\mathrm{g}$. This resulted in the following
Table 2. $\mathrm{CMC}_{90} \mathrm{MC}$ formulations tested in the subcutaneous biocompatibility study.

\begin{tabular}{|c|c|}
\hline Groups & APS and TEMED $(\mathrm{mM})$ \\
\hline $\mathrm{CMC}_{90} \mathrm{MC}-0$ & 0 \\
\hline $\mathrm{CMC}_{90} \mathrm{MC}-10$ & 10 \\
\hline $\mathrm{CMC}_{90} \mathrm{MC}-20$ & 20 \\
\hline
\end{tabular}

groups: $\mathrm{CMC}_{90} \mathrm{MC}-0$ (0 mM), $\mathrm{CMC}_{90} \mathrm{MC}-10$ (10 mM) and $\mathrm{CMC}_{90} \mathrm{MC}-20(20 \mathrm{mM})$ (Table 2). The $\mathrm{CMC}_{90} \mathrm{MC}-0$ hydrogels lacking redox initiators served as negative controls, and gelled in situ purely by thermogelation and were not covalently crosslinked. Subcutaneous injection in Sprague Dawley rats is routinely used to characterize the foreign body reaction to polymeric biomaterials, and the size of the animal allows for clinically-relevant injection volumes (500-1000 $\mu \mathrm{L})$ to be tested (Ibim et al., 1998; Wallace et al., 1992), as a prelude to evaluation in a large animal disc injury model. A $500 \mu \mathrm{L}$ volume of the sterilized hydrogel solution was injected subcutaneously with a $20 \mathrm{G}$ needle into each rat, at four sites on the dorsum, while under isoflurane- $\mathrm{O}_{2}$ general anesthesia, in compliance with a protocol approved by the Institutional Animal Care \& Use Committee of The City College of New York. The rats were transferred back to their cages 30 min after injection to ensure adequate gelation of the polymer solution. They were fed a normal diet and monitored for changes in behavior and infection at the injection sites. At day 30, the animals were euthanized by $\mathrm{CO}_{2}$ asphyxiation, after which, the hydrogels were excised with the surrounding fibrous capsule intact and the foreign body response was assessed using histological methods. The study was conducted in accordance with ISO standard 109936:2007, to test for local effects of medical devices after implantation.

The isolated hydrogel samples were fixed in zinc buffered formalin and processed in EGME, followed by a second dehydration step using 2-propanol for $5 \mathrm{~h}$. The specimens were cleared using methyl salicylate for $10 \mathrm{~h}$ and infiltrated with paraffin for $6 \mathrm{~h}$, after which the samples were embedded in paraffin. Sections, 5-8 $\mu \mathrm{m}$ in thickness, were prepared using a Microm Rotary Microtome (Model HC 325; Thermo Scientific, Walldorf, Germany). Sections were deparaffinized using petroleum ether followed by EGME rinses. After hydration, the sections were stained with hematoxylin and eosin (H\&E) (Sigma-Aldrich), alcian blue (SigmaAldrich) and picrosirius red (Polysciences, Inc., Warrington, PA). A polarized filter was used to view the picrosirius-red-stained samples to identify the birefringent collagenous capsules. For CD68 staining, deparaffinized and hydrated sample sections underwent heat-induced antigen retrieval in citrate buffer, and were stained with a mouse anti-CD68 antibody (Abcam, Cambridge, MA) (1:400 dilution) with a DAB-based chromogen to detect macrophages. In addition to non-immune IgG (Sigma-Aldrich) 
controls, rat spleen sections were used as positive controls. The images were captured using a Zeiss Axio Imager Z1 (Carl Zeiss, USA) optical microscope. The fibrous capsule thickness was measured using ImageJ (NIH) (Schneider et al., 2012).

\section{Results}

Spectral analysis of modified CMC and MC polymers using ${ }^{1} \mathrm{H}-\mathrm{NMR}$ revealed a methacrylation modification of $15 \%$ and $8 \%$, respectively (Fig. 1a). Preliminary assessment of MC polymer solutions at varying macromer concentrations demonstrated that a minimum of $3 \%(\mathrm{w} / \mathrm{v})$ was required to create a thermogelling network of $\mathrm{MC}$ at $37^{\circ} \mathrm{C}$. Therefore, all formulations of CMC were combined with a $3 \%$ (w/v) MC hydrogel solution. Gross observation of the samples $30 \mathrm{~min}$ post injection suggested that a minimum of $20 \mathrm{mM}$ redox initiators APS and TEMED, and a minimum of $3 \%(\mathrm{w} / \mathrm{v})$ CMC are required to provide consistent gelation and stable hydrogel formation within bovine motion segments. Additional evaluation showed that the increased viscosity of the methacrylated CMC-MC DPN compared to methacrylated CMC alone allowed for local retention of the injected material and subsequent curing in situ (Fig. 1b,c). Thus, two formulations at $6 \%(\mathrm{w} / \mathrm{v})$ of total polymer, $\mathrm{CMC}_{90} \mathrm{MC}(\mathrm{CMC}(90 \mathrm{kDa}$, $3 \%(\mathrm{w} / \mathrm{v}))+\mathrm{MC}(3 \%(\mathrm{w} / \mathrm{v}))$ and $\mathrm{CMC}_{250} \mathrm{MC}(\mathrm{CMC}$ $(250 \mathrm{kDa}, 3 \%(\mathrm{w} / \mathrm{v}))+\mathrm{MC}(3 \%(\mathrm{w} / \mathrm{v}))$, were chosen for material characterization using redox initiators at concentrations of $20 \mathrm{mM}$.

\section{Material characterization of in situ crosslinked hydrogels}

Material properties of CMC-MC hydrogels formed in situ within bovine motion segments were evaluated. Initial rheological assessment of the methacrylated $\mathrm{CMC}_{90}$ and $\mathrm{CMC}_{250}$ base polymers highlighted the differences between their viscosities. The complex viscosity at $37{ }^{\circ} \mathrm{C}$ of $\mathrm{CMC}_{90}(0.02 \pm 0.01 \mathrm{~Pa} \times \mathrm{s})$ was significantly lower than $\mathrm{CMC}_{250}(0.32 \pm 0.06 \mathrm{~Pa} \times \mathrm{s})$. Nevertheless, the compressive mechanical properties of the crosslinked $\mathrm{CMC}_{90} \mathrm{MC}$ hydrogels following in situ gelation were better than those of the $\mathrm{CMC}_{250} \mathrm{MC}$ hydrogels, with a significantly higher equilibrium Young's modulus $\left(E_{y}\right)$ of $16.62 \pm 3.59 \mathrm{kPa}$ and a significantly lower \% relaxation of $33.82 \pm 3.11$ compared to the $\mathrm{CMC}_{250} \mathrm{MC}$ gels $(8.249 \pm 1.372 \mathrm{kPa}$ and $41.708 \pm 0.048$, respectively) (Fig. 3). Furthermore, the $E_{y}(34.27 \pm 4.53 \mathrm{kPa})$ of $\mathrm{CMC}_{90} \mathrm{MC}$ hydrogels formed in vitro in a custom casting device, was significantly higher than that of hydrogels formed in situ within motion segments. No significant differences were noted between the $Q_{w}$ of hydrogels from $\mathrm{CMC}_{90} \mathrm{MC}$ and $\mathrm{CMC}_{250} \mathrm{MC}$ formulations. Both formulations had an average $Q_{w}$ of $\approx 25$ (Fig. 3c).

Based on the lower complex viscosity and $E_{y^{\prime}}$ the $\mathrm{CMC}_{90} \mathrm{MC}$ formulation was further characterized. Rheometry at $37{ }^{\circ} \mathrm{C}$ revealed a gelation time of $3.96 \pm 0.21 \mathrm{~min}$ (Fig. 4a) and a complex viscosity of $440.7 \pm 166.4 \mathrm{~Pa} \times \mathrm{s}$ within $30 \mathrm{~s}$ upon loading the hydrogel on the instrument.

\section{Motion segment biomechanics}

Biomechanical behavior of the motion segments was evaluated using a repeated measures design, where samples underwent testing under three conditions: Intact, Injured (post discectomy) and Implanted (with $\mathrm{CMC}_{90} \mathrm{MC}$ hydrogel). Gross observations of the bovine motion segments, upon completion of the mechanical testing regimen, revealed that the $\mathrm{CMC}_{90} \mathrm{MC}$ hydrogel implant filled the void NP region of the IVD (Fig. 4b). Changes in the IVD structure were visualized with toluidine blue staining of histological sections of motion segments under the three conditions: Intact, Injured and Implanted (Fig. 4c). The Injured samples exhibited a distinct void space as a result of the lost NP, while in the Implanted samples of the Experimental group, the $\mathrm{CMC}_{90} \mathrm{MC}$ hydrogel filled this void and interdigitated with the surrounding tissue.

\section{Effect of discectomy}

Injury by discectomy of motion segments, in both Experimental and Sham groups, resulted in significant changes in all measured biomechanical parameters, except for the compressive and tensile stiffness in the Sham group that did not exhibit a significant effect post injury (Fig. 5f-g). Representative force-displacement curves for both the Experimental
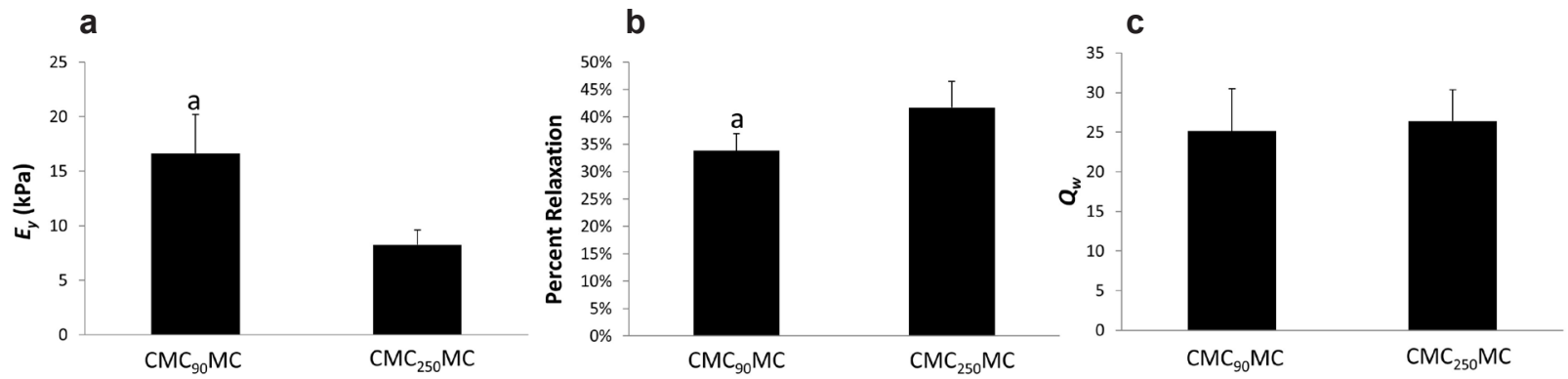

Fig. 3. Material properties of $\mathrm{CMC}_{90} \mathrm{MC}$ and $\mathrm{CMC}_{250} \mathrm{MC}$ hydrogels created in situ within bovine motion segments. (a) Equilibrium Young's Modulus $\left(E_{y}\right)$. (b) Percent relaxation (c) Equilibrium swelling ratio $\left(Q_{w}\right)$. a Significantly different w.r.t. $\mathrm{CMC}_{250} \mathrm{MC}$ hydrogels. 
a

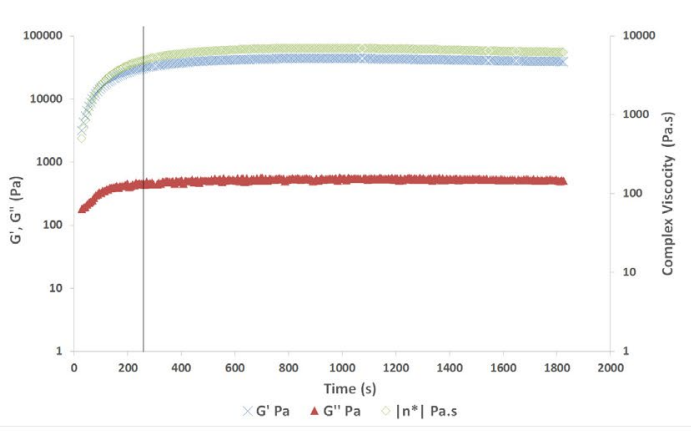

b

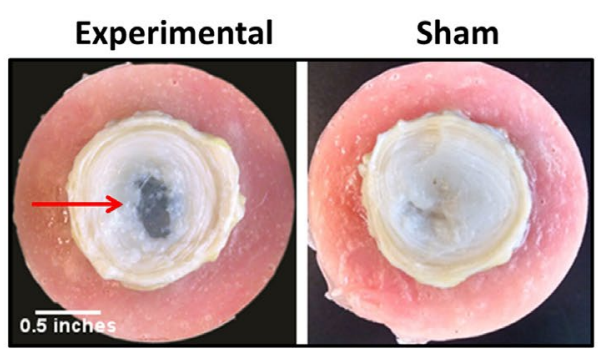

C

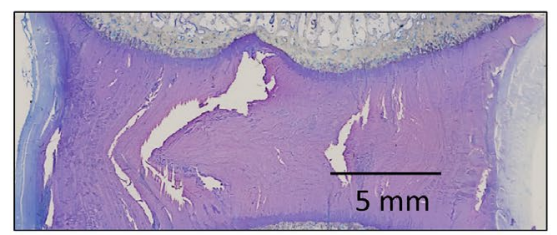

Intact

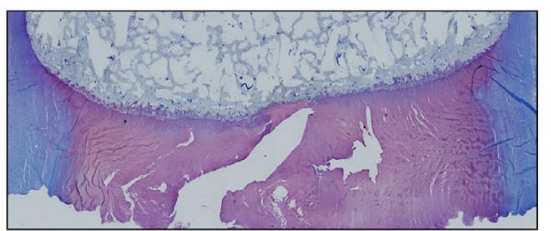

Implanted

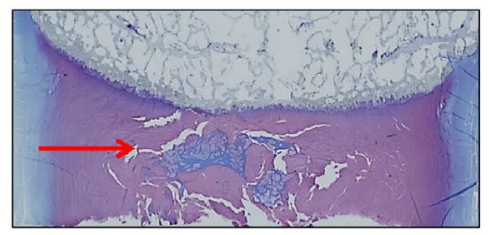

Fig. 4. Gelation and post-injection characteristics of the $\mathrm{CMC}_{90} \mathrm{MC}$ hydrogel. (a) Gelation kinetics of the $\mathrm{CMC}_{90} \mathrm{MC}$ hydrogel represented by $\mathrm{G}^{\prime}, \mathrm{G}^{\prime \prime}$ and complex viscosity with time. Black line represents the point of gelation completion. (b) Gross images illustrating the presence of the $\mathrm{CMC}_{90} \mathrm{MC}$ hydrogel completely filling the NP void in the Experimental group contrasted with the void NP in the Sham group. (c) Histological sections of motion segments in the Intact, Injured and Implanted conditions stained with toluidine blue. Red arrow indicates the $\mathrm{CMC}_{90} \mathrm{MC}$ hydrogel filling the $\mathrm{NP}$ void space.

and Sham groups are shown in Figs. 5a and 5b, respectively, displaying the effect of discectomy and hydrogel treatment on the curve profiles. In the Experimental group, the ROM of the injured samples increased by $15.5 \%$, from $2.92 \pm 0.23 \mathrm{~mm}$ in intact to $3.37 \pm 0.43 \mathrm{~mm}$ in injured specimens (Fig. $5 \mathrm{c}$ ). Discectomy also resulted in a significant reduction in the NZ stiffness, from $0.013 \pm 0.01 \mathrm{kN} / \mathrm{mm}$ in Intact to $0.003 \pm 0.007 \mathrm{kN} / \mathrm{mm}$ in Injured samples (Fig. $5 \mathrm{~d}$ ). This was accompanied by a significant increase in the NZ length and slow ramp stiffness, from $1.73 \pm 0.1 \mathrm{~mm}$ to $2.26 \pm 0.21 \mathrm{~mm}$ (Fig. 5e) and $0.22 \pm 0.06 \mathrm{kN} /$ $\mathrm{mm}$ to $0.25 \pm 0.06 \mathrm{kN} / \mathrm{mm}$ (Fig. $5 \mathrm{~h}$ ), respectively. Furthermore, injured motion segments suffered a $\approx 20 \%$ drop in IVD height compared to Intact IVD height $(7.68 \pm 1.34 \mathrm{~mm}$ ) (Fig. $6 \mathrm{~d})$, which can also be visually observed in the x-ray radiographs (Fig. 6a,b). No significant differences were observed in Intact and Injured conditions between the Experimental and Sham groups (Fig. 6).

\section{Effect of hydrogel treatment}

The $\mathrm{CMC}_{90} \mathrm{MC}$ hydrogel did not extrude from the motion segments at any point during the axial mechanical testing or incubation. Upon injection, the implant significantly reduced the ROM in the Experimental group from $3.37 \pm 0.43 \mathrm{~mm}$ in Injured to $2.76 \pm 0.35 \mathrm{~mm}$ in Implanted specimens, thus restoring the ROM back to the Intact value $(2.92 \pm 0.23 \mathrm{~mm})$ (Fig. 5c). The NZ stiffness of Implanted samples in the Experimental group was $0.02 \pm 0.01 \mathrm{kN} / \mathrm{mm}$, which significantly exceeded the Injured condition
$(0.003 \pm 0.007 \mathrm{kN} / \mathrm{mm})$, and recovered to Intact values $(0.012 \pm 0.009 \mathrm{kN} / \mathrm{mm})$ (Fig. 5d). After hydrogel implantation, the compressive and slow-ramp stiffnesses significantly decreased compared to the Injured group, measuring $0.50 \pm 0.13 \mathrm{kN} / \mathrm{mm}$ and $0.21 \pm 0.05 \mathrm{kN} / \mathrm{mm}$, respectively (Fig. $5 \mathbf{f}, \mathbf{h}$ ), while the tensile stiffness, $0.19 \pm 0.04 \mathrm{kN} / \mathrm{mm}$ (Fig. 5g), was significantly higher than the Injured specimens. Additionally, the NZ length of the Experimental group was $1.59 \pm 0.21 \mathrm{~mm}$ after hydrogel implantation, and was similar to Intact samples $(1.71 \pm 0.1 \mathrm{~mm})$, while it maintained a high value of $2.23 \pm 0.38 \mathrm{~mm}$, similar to Injured $(2.25 \pm 0.38 \mathrm{~mm})$ in the Sham group (Fig. 5e). Thus, all biomechanical parameters of the Implanted condition in the Experimental group were restored to the Intact condition. Conversely, most of the biomechanical properties in the 'No Implant' condition of the Sham group were significantly different from the Intact condition and similar to the Injured condition, with the exception of compressive and tensile stiffness, which did not show significant differences between the Intact and 'No Implant' conditions. Under the Implanted condition, restoration of the above biomechanical parameters was accompanied by recovery of IVD height to values in intact motion segments. The Experimental group exhibited no significant differences between Intact $(7.76 \pm 1.48 \mathrm{~mm})$ and Implanted specimens $(7.72 \pm 0.87 \mathrm{~mm})$, while the IVD height of 'No Implant' samples $(6.19 \pm 1.18 \mathrm{~mm})$ of the Sham group was not significantly different from Injured motion segments $(6.01 \pm 1.11 \mathrm{~mm})$ (Fig. 6d) 
口Intact $\square$ Injured $\square$ Implanted/No implant
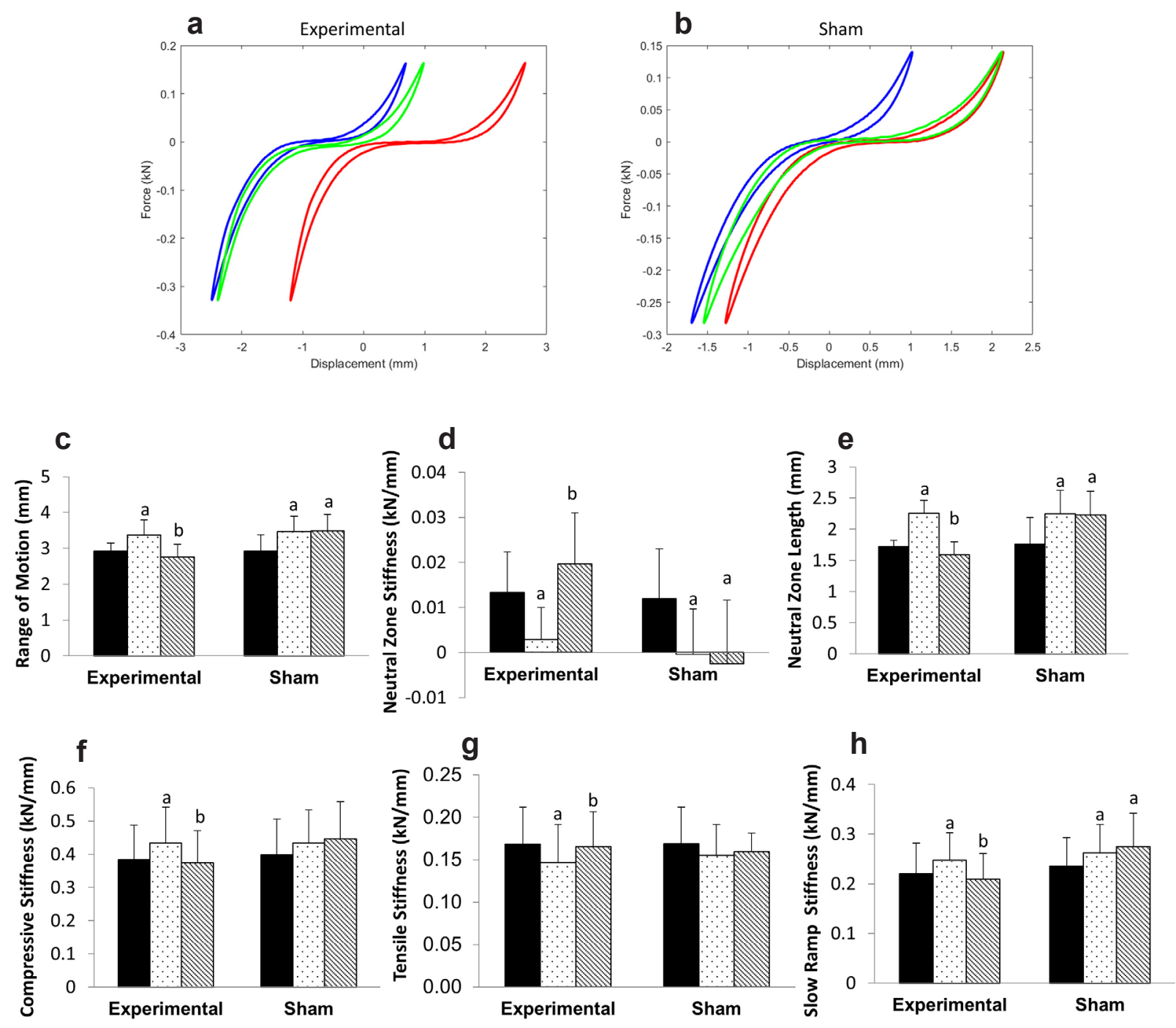

Intact Injured $\quad$ Implanted/No implant

Fig. 5. Biomechanical evaluation of motion segments with and without hydrogel repair. (a,b) Representative force-displacement curves of motion segment samples over the three-day test from the Experimental and Sham groups. Comparison of biomechanical parameters between Experimental and Sham groups in the Intact, Injured and Implanted/No Implant conditions (c) Range of motion (d) Neutral zone stiffness (e) Neutral zone length (f) Compressive stiffness (g) Tensile stiffness (h) Slow ramp stiffness. ${ }^{a}$ Significantly different w.r.t intact, ${ }^{\mathrm{b}}$ Significantly different w.r.t injured $p<0.05$.

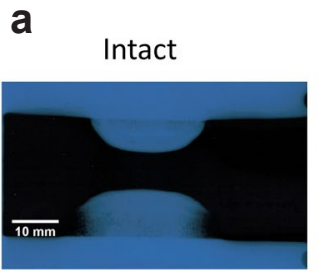

b
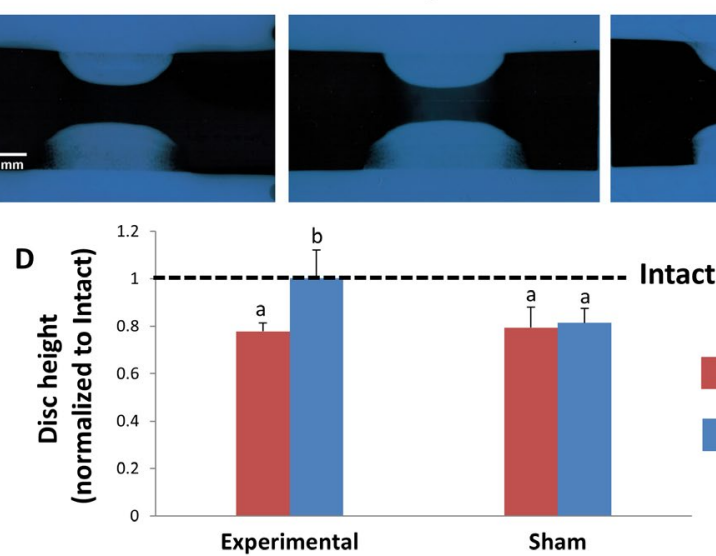

Fig. 6. Changes in IVD height between Intact, Injured and Implanted conditions. $(\mathbf{a}, \mathbf{b}, \mathbf{c})$. $X$-ray radiographs of explants at the three conditions. Red arrow in (c) indicates the presence of the $\mathrm{CMC}_{90} \mathrm{MC}$ hydrogel inside the implanted motion segment. (d) Numerical values of the IVD height were obtained from the radiographs, which were normalized to Intact IVD height values (dotted line). ${ }^{a}$ Significantly different w.r.t intact, ${ }^{b}$ Significantly different w.r.t injured $p<0.05$ 


\section{Biological response}

The cytocompatibility of the $\mathrm{CMC}_{90} \mathrm{MC}$ hydrogels used in this study, created with $20 \mathrm{mM}$ of APS and TEMED, was tested in vitro on encapsulated cells over $6 \mathrm{~d}$ in culture. PicoGreen analysis of the DNA content in the gels showed no significant differences on day 1 and 6 between the $\mathrm{CMC}_{90} \mathrm{MC}$ hydrogels and the control CMC gels fabricated using $10 \mathrm{mM}$ redox initiators, a formulation tested in previous cell-based studies (Varma, 2016). Further, qualitative assessment of cell viability by Live/Dead staining revealed that most of the cells were alive in both groups and were visually comparable through day 6 (Fig. 7).

The effect of redox initiator concentrations on the biocompatibility of the CMC-MC hydrogels was evaluated by comparing subcutaneously formed hydrogels with $0\left(\mathrm{CMC}_{90} \mathrm{MC}-0\right), 10 \mathrm{mM}$ $\left(\mathrm{CMC}_{90} \mathrm{MC}-10\right)$ and $20 \mathrm{mM}\left(\mathrm{CMC}_{90} \mathrm{MC}-20\right)$ redox initiators. After $30 \mathrm{~d}$ of subcutaneous implantation in vivo, the rats had grown in size, displayed normal sleeping and eating habits, and exhibited no redness or inflammation at the injection sites. Overall, the animals appeared healthy and maintained normal mobility and behavior. The harvested $\mathrm{CMC}_{90} \mathrm{MC}-10$ and $\mathrm{CMC}_{90} \mathrm{MC}-20$ hydrogels were surrounded by fibrous capsules $111.29 \pm 44.59 \mu \mathrm{m}$ and $77.76 \pm 28.45 \mu \mathrm{m}$ thick, respectively. The $\mathrm{CMC}_{90} \mathrm{MC}-0$ hydrogels did not reveal a distinct capsule and unlike the crosslinked gels, displayed cellularity and random arrangement of fibrous tissue within the hydrogel (Fig. 8a-c). These uncrosslinked samples were also too mechanically weak to handle and exhibited limited presence of $\mathrm{CMC}$, as indicated by minimal alcian blue staining of the gels compared to the covalently crosslinked $\mathrm{CMC}_{90} \mathrm{MC}-10$ and $\mathrm{CMC}_{90} \mathrm{MC}-20$ hydrogels, which stained intensely blue and maintained their shape. Additionally, collagen fibers were highlighted by picrosirius red and were primarily limited to the fibrous capsule around the crosslinked hydrogels, while they were found randomly arranged throughout the $\mathrm{CMC}_{90} \mathrm{MC}-0$ samples (Fig. 8a-f). Furthermore, CD68 staining of the $\mathrm{CMC}_{90} \mathrm{MC}-10$ and $\mathrm{CMC}_{90} \mathrm{MC}-20$ hydrogels exhibited a layer of macrophages adjacent to the hydrogel implants within the fibrous capsule, while the $\mathrm{CMC}_{90} \mathrm{MC}-0$ hydrogels showed the presence of stained cells distributed within the diffuse material (Fig. 8g-1). The size and shape of the gels remained stable over the 30-day in vivo study based on the gross appearance of the implants (Fig. 8m-o).
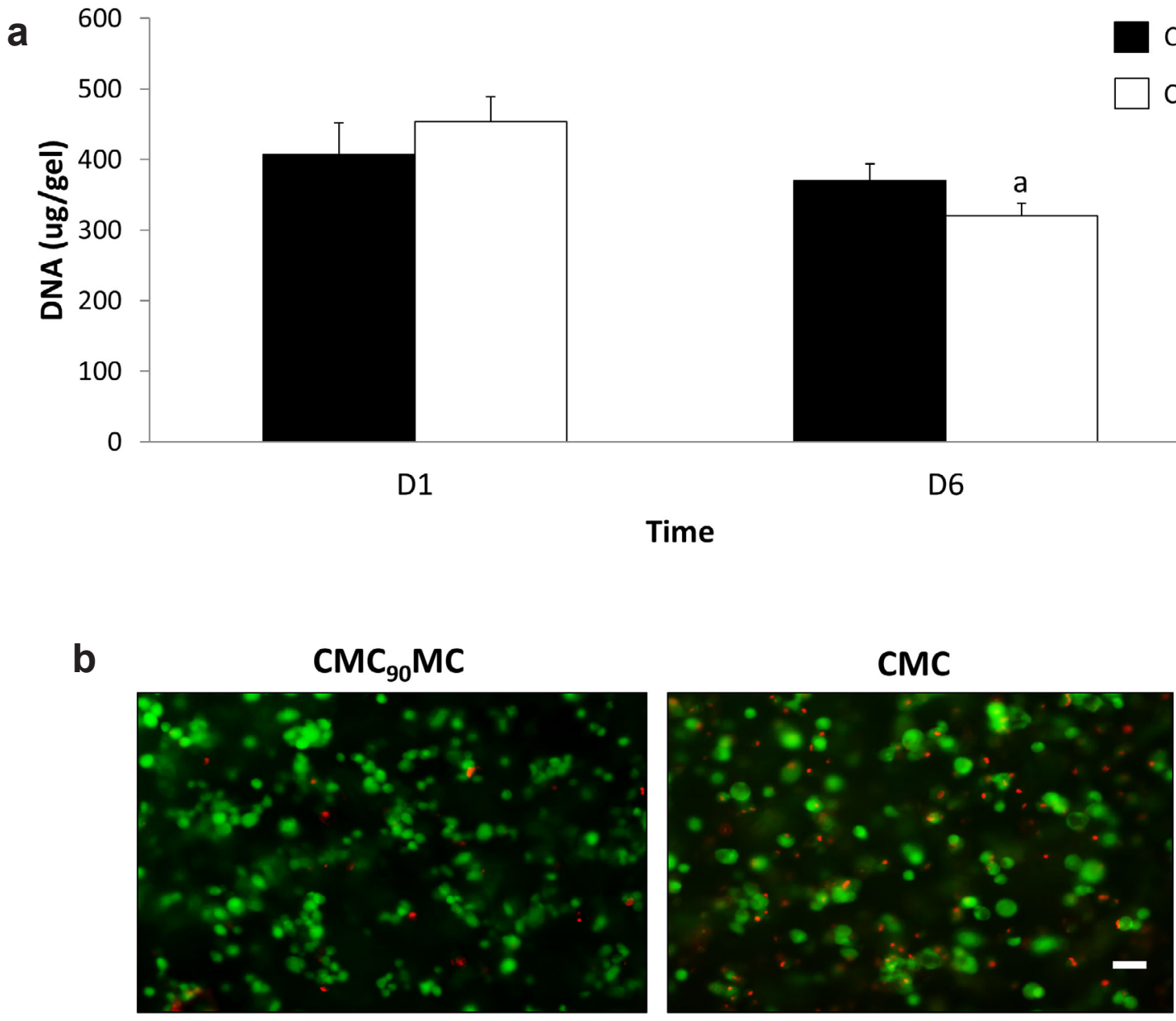

Fig. 7. Cytotoxicity assessment of the $\mathrm{CMC}_{90} \mathrm{MC}$ hydrogel formulation. (a) DNA measurements quantified using PicoGreen on day 1 and day 6 in $\mathrm{CMC}_{90} \mathrm{MC}$ and $\mathrm{CMC}$ hydrogels. ${ }^{a}$ Significantly different w.r.t to day 1 (b) Live/Dead staining of the $\mathrm{CMC}_{90} \mathrm{MC}$ and $\mathrm{CMC}$ hydrogels on day 6, live cells stained in green and dead cells stained in red. Scale bar $=50 \mu \mathrm{m}$. 

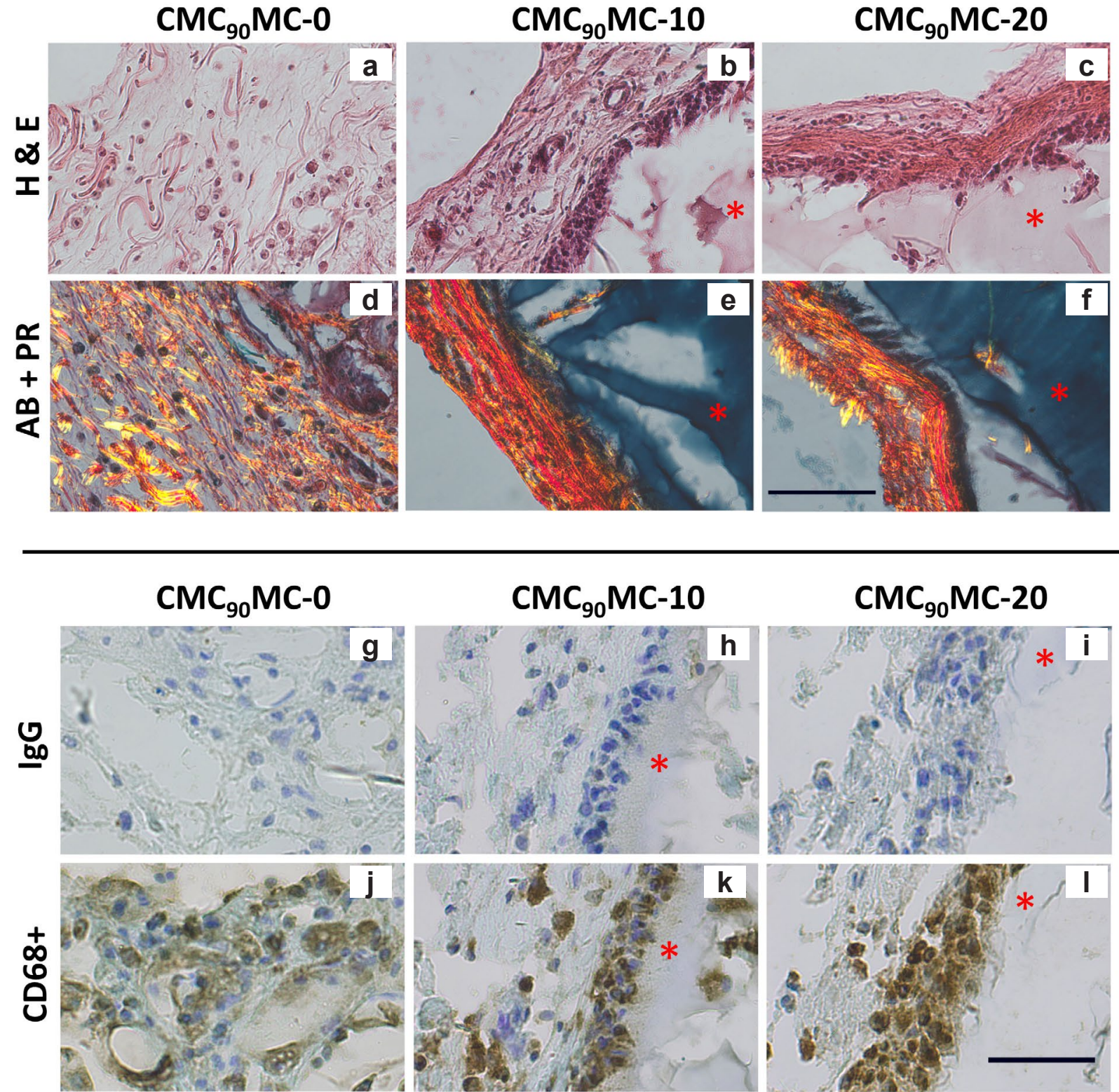

Day 0

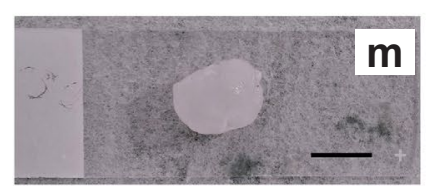

Day 7

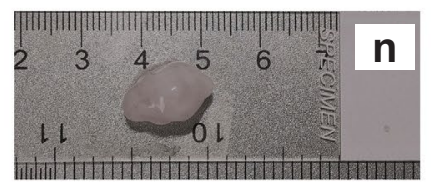

Day 30

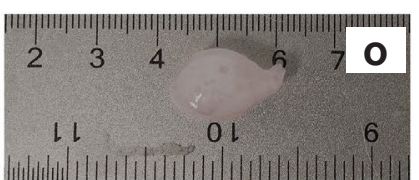

Fig. 8. Top panel shows histological staining comparing the fibrous capsule thickness and composition in $\mathrm{CMC}_{90} \mathrm{MC}-0, \mathrm{CMC}_{90} \mathrm{MC}-10$ and $\mathrm{CMC}_{90} \mathrm{MC}-20$ hydrogels isolated $30 \mathrm{~d}$ after subcutaneous injection in vivo. (a-c) H\&E images depicting the fibrous capsule and the adjacent hydrogel. (d-f) Alcian blue (AB) stains the $\mathrm{CMC}_{90} \mathrm{MC}$ hydrogel while the picrosirius red (PR) highlights the collagen fibers in the fibrous capsule. Minimal blue staining indicates the remnants of the hydrogel in the $\mathrm{CMC}_{90} \mathrm{MC}-0$ sample $(\mathbf{a}, \mathbf{d})$. The hydrogel is indicated by a red asterisk $(*)$. Scale bar $=100 \mu \mathrm{m}$. Bottom panel displays immunohistochemistry staining of implants isolated at one month. (g), (h) and (i) are the IgG controls for $\mathrm{CMC}_{90} \mathrm{MC}-0, \mathrm{CMC}_{90} \mathrm{MC}-10$ and $\mathrm{CMC}_{90} \mathrm{MC}-20$, respectively. Staining demonstrating the presence of $\mathrm{CD} 68+$ macrophages in (j) $\mathrm{CMC}_{90} \mathrm{MC}-0$, (k) $\mathrm{CMC}_{90} \mathrm{MC}-10$ and (1) $\mathrm{CMC}_{90} \mathrm{MC}-20$ hydrogels. The hydrogel is stained blue with toluidine blue and is indicated by a red asterisk ${ }^{*}$ ). Scale bar $=50 \mu \mathrm{m}$. (m-o) Gross images of $\mathrm{CMC}_{90} \mathrm{MC}-20$ hydrogels isolated from the subcutaneous pouch of rats at different time points in the $30 \mathrm{~d}$ study. Scale bar $=10 \mathrm{~mm}$. 


\section{Discussion}

There is a great need for NP replacement materials, since current treatments for degeneration with herniation commonly involve discectomy, which can cause loss of IVD height, alter IVD biomechanics and advance degeneration. This is the first study to report the development of a redox-initiated, thermogelling, crosslinked DPN of methacrylated CMC and MC. This novel DPN was injectable, gelled in situ, filled irregularly shaped voids, approximated native NP material properties, and restored the biomechanics of the IVD to healthy levels following discectomy.

\section{Polymer selection and hydrogel development}

In situ gelation characterization of iterative formulations - prepared by varying the molecular weight of CMC, macromer concentration of methacrylated CMC and the redox initiator concentration - revealed two potential candidates as NP replacements: $\mathrm{CMC}_{90} \mathrm{MC}$ and $\mathrm{CMC}_{250} \mathrm{MC}$. Since the $\mathrm{CMC}_{250} \mathrm{MC}$ hydrogels were composed of a higher molecular weight $\mathrm{CMC}$, they were expected to have superior mechanical properties compared to the $\mathrm{CMC}_{90} \mathrm{MC}$ hydrogels. Surprisingly, the $\mathrm{CMC}_{90} \mathrm{MC}$ group had greater compressive modulus and lower percent relaxation following in situ gelation within the bovine IVD. The lower complex viscosity of $\mathrm{CMC}_{90}$ likely allowed for more uniform mixing of the $\mathrm{CMC}$ and $\mathrm{MC}$ polymers with the redox initiators; therefore, resulting in improved crosslinking and mechanical properties of the DPN. Thus, $\mathrm{CMC}_{90} \mathrm{MC}$ was chosen to be further tested for its potential to restore biomechanics of injured bovine motion segments.

The injectability of the $\mathrm{CMC}_{90} \mathrm{MC}$ solution may be ascertained by measurement of its complex viscosity $\left(\eta^{*}\right)(440.7 \pm 166.4$ Pa s), which was obtained within the first 30 s upon loading the hydrogel on the rheometer. This value is on the same order of magnitude as the $\eta^{*}$ of injectable dermal fillers available commercially, which range between 58-1199 Pa s (Falcone and Berg, 2008). Rheological analyses also determined the gelation time $(3.96 \pm 0.21 \mathrm{~min})$ at $37{ }^{\circ} \mathrm{C}$ to be in accordance with the ISO standard 5833/1-1999 E for injectable materials (4-15 min), which is a distinct advantage over other in situ gelling systems that require several hours to achieve maximum mechanical properties (Smith et al., 2014). The lower viscosity of the pre-gelled $\mathrm{CMC}_{90} \mathrm{MC}$ solution allowed penetration of the material into the cracks and fissures of the NP and AF, in addition to filling of the larger void spaces. However, histological analysis revealed incomplete filling of the smaller voids within the NP. While these smaller cavities could be artifacts from histological processing, this observation suggested that the infiltration of the hydrogel solution may be limited in regions of reduced connectivity, such as those likely associated with smaller voids. Future assessment may include microcomputed tomography measurements of the radiopaque hydrogel post injection to quantify filling of the NP deficit (Gullbrand et al., 2017).

Comparing the mechanical properties of $\mathrm{CMC}_{90} \mathrm{MC}$ hydrogels formed in vitro, versus in situ revealed that the confined area of a casting device prevented loss of free radicals compared to the in situ environment of the NP void space, resulting in a significantly higher $E_{y}$ of gels formed in vitro. The $E_{y}$ values obtained by unconfined compression testing of both $\mathrm{CMC}_{90} \mathrm{MC}$ and $\mathrm{CMC}_{250} \mathrm{MC}$ gels formed in situ were higher than that reported for the native human NP tissue ( $E_{y}$ of $\approx 5 \mathrm{kPa}$ ) (Cloyd et al., 2007), although degeneration-related changes are expected to increase native tissue values (Umehara et al., 1996). Furthermore, the CMC-MC hydrogel has an equilibrium compressive modulus similar to other NP replacement materials, which range between 9-35 kPa (Frith et al., 2013; Smith et al., 2014; Thomas et al., 2010). Hydrogels of greater compressive strength might be necessary to withstand the loads experienced in injured or degenerated IVDs in vivo. Additionally, the $\mathrm{CMC}_{90} \mathrm{MC}$ hydrogels are expected to degrade hydrolytically in vivo due to hydrolysis of interchain ester crosslinks (Gupta and Nicoll, 2014; Reza and Nicoll, 2010; Varma et al., 2014), potentially reducing their mechanical strength over time. As such, measuring the stability and degradation kinetics of these formulations in vivo would be an essential goal for future studies.

\section{Effect of discectomy and hydrogel implantation}

Axial biomechanical behaviors of the motion segment were evaluated to detect the loss of NP pressurization and changes in the neutral zone (NZ), a parameter that describes the region of the IVD presenting minimal resistance to load; and, thereby, the portion of the force-deflection curve most sensitive to loss of pressurization due to NP degeneration or injury (Iatridis et al., 2013; Panjabi, 1992). The effect of discectomy on bovine IVD motion segments was prominently demonstrated by the divergence of all biomechanical parameters in most of injured samples when compared to their intact values. The degree of injury in the motion segments was clinically relevant, as indicated by $\approx 20 \%$ loss of IVD height post discectomy that is comparable to the $25 \%$ disc height loss observed in human subjects undergoing discectomy (McGirt et al., 2009). The ROM of all injured motion segments rose significantly from their intact values. As expected, the loss of the NP dramatically impacted the NZ, demonstrated by the significant reduction in NZ stiffness and increase in NZ length. Although the compressive and tensile stiffnesses of samples in the Experimental group changed significantly after discectomy, this effect was not consistent in the samples of the Sham group. This result could be attributed to the dependence of compressive and tensile stiffnesses predominantly on AF integrity (Johannessen et al., 2006). The depressurization and AF destabilization during discectomy likely resulted 
in the significant reduction in tensile stiffness in the Experimental group, as reported previously (Likhitpanichkul et al., 2014). Further, discectomy significantly increased compressive and slow ramp stiffness in the Experimental group, which suggested the transfer of load to the stiffer AF component of the IVD or to the cartilaginous endplates.

The IVD height is an important clinical parameter and an indicator of the level of hydration and hydrostatic pressure in the IVD. Thus, it was promising to observe that the injured IVDs regained their intact IVD heights after injection of the $\mathrm{CMC}_{90} \mathrm{MC}$ hydrogel. Moreover, all measured biomechanical parameters (ROM, NZ length, and slow ramp, compressive, tensile and NZ stiffnesses) were restored to intact values upon hydrogel injection, while the Sham samples showed no improvements from their injured values. Although transannular discectomy has been found to measurably alter the compressive and tensile moduli, these parameters are often not restored using an NP replacement alone (Balkovec et al., 2013; Malhotra et al., 2012; Smith et al., 2014). Therefore, the recovery of these parameters with the $\mathrm{CMC}_{90} \mathrm{MC}$ hydrogel in the current study was unique but must be interpreted with some qualification since the Sham group displayed no statistically significant effect of discectomy on the compressive and tensile moduli.

Assessment of the NZ properties allows preferential measurement of the NP functionality in the IVD and is an important consideration for NP replacements. As expected, the NZ stiffness and length were restored back to Intact values with the hydrogel implant. Slow-ramp stiffness is proposed to reflect the mechanical response of the NP at low loads and low frequency (Johannessen et al., 2006). In the current study, the removal of the NP reduced the viscous contribution to the low-load response region of the IVD and transferred the compressive loads to the surrounding AF and endplates, resulting in an increase of the slow ramp stiffness. Replacement of the lost NP with the $\mathrm{CMC}_{90} \mathrm{MC}$ hydrogel restored the hydrated, viscous state of the NP, lowering slowramp stiffness values.

This initial biomechanical study aimed to test the functional capacity of the $\mathrm{CMC}_{90} \mathrm{MC}$ hydrogel under axial compression and tension, yet there were some limitations that should be noted. The repeated measures design that was employed helped reduce the variability between samples originating from different bovine tails. However, this design also limited the direct comparison of hydrogel-implanted discs with their 'No Implant' counterparts. Past preclinical and clinical studies demonstrate the susceptibility of NP replacements to fail under physiological loading, especially in the lower back under bending and torsion (Panjabi and White, 1980). Thus, even though axial compression is a primary loading mode in the NP of the human IVD, additional evaluation of the hydrogel in other degrees of freedom, such as in bending, is essential
- together with fatigue loading studies to assess resistance of the $\mathrm{CMC}_{90} \mathrm{MC}$ implant to herniation. Finally, although mechanical restoration of the NP is crucial to restrict progression of IVD degeneration, it is noteworthy that one of the primary failure modes of older generations of NP replacements has been by the annular injury post discectomy. Therefore, despite the fact that CMC-MC solutions can infiltrate into tissue cracks and fissures, including into AF tears surrounding the NP, parallel development of annular sealants or closure devices (Guterl et al., 2013; Likhitpanichkul et al., 2014; Parker et al., 2016) may be important to retain this or other NP replacement biomaterials, should future rigorous biomechanical testing show evidence for herniation.

\section{Biological response to redox-polymerized cellulosic hydrogels}

Independently, CMC and MC are established biocompatible polymers that are used in biomedical research and have an excellent safety profile with the US Food and Drug Administration (Miyamoto et al., 1989). The biological response assessed in the current study aimed at monitoring the toxic effects of the redox initiators necessary to create stable, crosslinked hydrogels in situ. The in vitro cytotoxicity study revealed adequate cytocompatibility of $\mathrm{CMC}_{90} \mathrm{MC}$ hydrogels, as quantified by DNA measurements, in comparison to the control group consisting of previously developed cytocompatible CMC hydrogels prepared with a lower redox initiator concentration of $10 \mathrm{mM}$ (Varma, 2016). The absence of a statistically significant increase in DNA content over time for both hydrogel formulations could be attributed to the limited proliferative capacity of anchorage-dependent fibroblasts in a hydrogel lacking binding sites (Guadagno and Assoian, 1991). The cytocompatibility results suggested the potential application of the DNP as a safe cell-delivery vehicle for biological repair of the NP, although additional evaluation of the material with more appropriate cell sources such as NP cells or mesenchymal stem cells would be necessary (Gullbrand et al., 2017; Gupta and Nicoll, 2015).

Implantation into the rodent subcutis is a widely accepted model to test the biological response to new materials, as specified in ISO standard 10993-6:2007. In addition, the subcutaneous pouch model was selected over a disc injection model since it is difficult to yield conclusive results from small animal IVD testing; as the injection volume into the IVD is very small, technically challenging and may not represent the limited nutrition condition of the human IVD in vivo environment. Photocrosslinked MC hydrogels, tested in a murine subcutaneous pouch model, reveal a subtle foreign body response with a $<80 \mu \mathrm{m}$ capsule size (Stalling et al., 2009). In the current study, the effect of redox initiator concentrations on the foreign body response to $\mathrm{CMC}_{90} \mathrm{MC}$ DPNs was studied by comparing the $\mathrm{CMC}_{90} \mathrm{MC}-20$ hydrogels with $\mathrm{CMC}_{90} \mathrm{MC}-10$ and $\mathrm{CMC}_{90} \mathrm{MC}-0$ samples in 
rats. The fibrous capsules observed around the $\mathrm{CMC}_{90} \mathrm{MC}-10$ and $\mathrm{CMC}_{90} \mathrm{MC}-20$ gels indicated a modest biological response with a capsule thickness of 70-150 $\mu \mathrm{m}$. This was comparable to responses seen with other biomaterials used for related biomedical applications (Bongio et al., 2013; Deng et al., 2010), and far less than the capsule thicknesses (>1000 $\mu \mathrm{m})$ associated with complications that require implant removal (Siggelkow et al., 2003). Additionally, the presence of macrophages observed in hydrogels with $\left(\mathrm{CMC}_{90} \mathrm{MC}-10\right.$ and $\left.\mathrm{CMC}_{90} \mathrm{MC}-20\right)$ and without redox initiators $\left(\mathrm{CMC}_{90} \mathrm{MC}-0\right)$ illustrated a normal foreign body response $30 \mathrm{~d}$ after implantation. With increasing implantation time, macrophages are typically found to recede, and the capsule becomes more defined, and exhibits aligned collagen and fibroblastic cells (Chiu et al., 2009; Kim et al., 2012).

Future biocompatibility testing in the subcutaneous space, with additional time points, will be necessary to observe the evolution of the initial biological response to redox-polymerized $\mathrm{CMC}_{90} \mathrm{MC}$ hydrogels. Qualitative assessment of the $\mathrm{CMC}_{90} \mathrm{MC}-20$ hydrogels, retrieved from the subcutaneous pouch of rats, revealed negligible differences in the size of the hydrogels over several points in the 30-day study. This observation indicated minimal degradation of the hydrogels over the duration of the study. However, with time, the hydrogels are expected to degrade hydrolytically due to cleavage of the interchain ester crosslinks. Unlike other biopolymers, CMC-MC formulations will not undergo enzymatic degradation in vivo. Future work will include in vitro hydrolytic degradation studies of the $\mathrm{CMC}_{90} \mathrm{MC}$ hydrogels over a longer time, and subcutaneous implantation investigations using ultrasound to measure implant volume changes to quantify degradation in vivo non-invasively (Wortsman et al., 2012). Finally, evaluation of the CMC-MC hydrogel in an in vivo large animal disc injury model will be necessary to examine its safety and biomechanical performance as an NP replacement material.

\section{Conclusions}

The injectable, redox-polymerized $\mathrm{CMC}_{90} \mathrm{MC}$ hydrogel developed in this study combined the compressive mechanical properties and polyanionic character of $\mathrm{CMC}$ with the intrinsic thermogelling nature of MC to create a unique crosslinked DPN NP replacement biomaterial. The CMC-MC hydrogel exhibited strong potential as an NP replacement that could be injected in a minimally invasive manner to restore IVD height and compressive biomechanical function post discectomy. The biocompatibility studies also motivate additional assessments to advance towards clinical translation. Future studies are required to evaluate herniation risk under bending and fatigue loading and to eventually determine if IVD repair with this biomaterial can limit disease progression when implanted in vivo.

\section{Acknowledgements}

This work was supported in part by NSF grants DMR 1207480 and IIP 1701120 (S.B.N.), and NIH Grant R01 AR057397 (J.C.I.). The authors would like to thank Damien Laudier at the City College of New York (CCNY) for technical assistance with histology, Dr. Padmanava Pradhan (CCNY) for guidance with ${ }^{1} \mathrm{H}-\mathrm{NMR}$ analysis, Nada Haq-Siddiqi (CCNY) for rheometry measurements to assess hydrogel gelation, and Phil Nasser (MSSM) and Carine Rognon (visiting research assistant at MSSM) for providing guidance and assistance with the explant biomechanics study.

\section{References}

Adams MA, McNally DS, Dolan P (1996) 'Stress' distributions inside intervertebral discs. The effects of age and degeneration. J Bone Joint Surg Br 78: 965972.

Alini M, Eisenstein SM, Ito K, Little C, Kettler AA, Masuda K, Melrose J, Ralphs J, Stokes I, Wilke HJ (2008) Are animal models useful for studying human disc disorders/degeneration? Eur Spine J 17: 2-19.

Balkovec C, Vernengo J, McGill SM (2013) The use of a novel injectable hydrogel nucleus pulposus replacement in restoring the mechanical properties of cyclically fatigued porcine intervertebral discs. J Biomech Eng 135: 61004-61005.

Becker A, Held H, Redaelli M, Strauch K, Chenot JF, Leonhardt C, Keller S, Baum E, Pfingsten M, Hildebrandt J, Basler HD, Kochen MM, DonnerBanzhoff N (2010) Low back pain in primary care: costs of care and prediction of future health care utilization. Spine (Phila Pa 1976) 35: 1714-1720.

Binetti VR, Fussell GW, Lowman AM (2014) Evaluation of two chemical crosslinking methods of poly(vinyl alcohol) hydrogels for injectable nucleus pulposus replacement. J Appl Polym Sci 131: 1-8.

Bongio M, Van Den Beucken JJJ, Nejadnik MR, Tahmasebi Birgani Z, Habibovic P, Kinard LA, Kasper FK, Mikos AG, Leeuwenburgh SCG, Jansen JA (2013) Subcutaneous tissue response and osteogenic performance of calcium phosphate nanoparticleenriched hydrogels in the tibial medullary cavity of guinea pigs. Acta Biomater 9: 5464-5474.

Cannella M, Isaacs JL, Allen S, Orana A, Vresilovic E, Marcolongo M (2014) Nucleus implantation: the biomechanics of augmentation versus replacement with varying degrees of nucleotomy. J Biomech Eng 136: 051001 doi: 10.1115/1.4027056.

Chiu YL, Chen SC, Su CJ, Hsiao CW, Chen YM, Chen HL, Sung HW (2009) pH-triggered injectable hydrogels prepared from aqueous N-palmitoyl chitosan: in vitro characteristics and in vivo biocompatibility. Biomaterials 30: 4877-4888.

Cloyd JM, Malhotra NR, Weng L, Chen W, Mauck RL, Elliott DM (2007) Material properties in unconfined compression of human nucleus pulposus, 
injectable hyaluronic acid-based hydrogels and tissue engineering scaffolds. Eur Spine J 16: 1892-1898.

Deng C, Zhang P, Vulesevic B, Kuraitis D, Li F, Yang AF, Griffith M, Ruel M, Suuronen EJ (2010) A collagen-chitosan hydrogel for endothelial differentiation and angiogenesis. Tissue Eng Part A 16: 3099-3109.

Di Martino A, Vaccaro AR, Lee JY, Denaro V, Lim MR (2005) Nucleus pulposus replacement: basic science and indications for clinical use. Spine (Phila Pa 1976) 30: S16-22.

Endean A, Palmer K, Coggon D (2011) Potential of MRI findings to refine case definition for mechanical low back pain in epidemiological studies: a systematic review. Spine (Phila Pa 1976) 36: 160-169.

Falcone SJ, Berg RA (2008) Crosslinked hyaluronic acid dermal fillers: a comparison of rheological properties. J Biomed Mater Res A 87: 264-271.

Frith JE, Cameron AR, Menzies DJ, Ghosh P, Whitehead DL, Gronthos S, Zannettino ACW, Cooper-White JJ (2013) An injectable hydrogel incorporating mesenchymal precursor cells and pentosan polysulphate for intervertebral disc regeneration. Biomaterials 34: 9430-9440.

Gan Y, Li P, Wang L, Mo X, Song L, Xu Y, Zhao C, Ouyang B, Tu B, Luo L, Zhu L, Dong S, Li F, Zhou Q (2017) An interpenetrating network-strengthened and toughened hydrogel that supports cell-based nucleus pulposus regeneration. Biomaterials 136: 12-28.

Gold GT, Varma DM, Taub PJ, Nicoll SB (2015) Development of crosslinked methylcellulose hydrogels for soft tissue augmentation using an ammonium persulfate-ascorbic acid redox system. Carbohydr Polym 134: 497-507.

Gold GT, Varma DM, Harbottle D, Gupta MS, Stalling SS, Taub PJ, Nicoll SB (2014) Injectable redoxpolymerized methylcellulose hydrogels as potential soft tissue filler materials. J Biomed Mater Res A 102: 4536-4544.

Guadagno TM, Assoian RK (1991) G1/S control of anchorage-independent growth in the fibroblast cell cycle. J Cell Biol 115: 1419-1425.

Gullbrand SE, Schaer TP, Agarwal P, Bendigo JR, Dodge GR, Chen W, Elliott DM, Mauck RL, Malhotra NR, Smith LJ (2017) Translation of an injectable tripleinterpenetrating-network hydrogel for intervertebral disc regeneration in a goat model. Acta Biomater 60: 201-209.

Gupta MS, Nicoll SB (2014) Functional nucleus pulposus-like matrix assembly by human mesenchymal stromal cells is directed by macromer concentration in photocrosslinked carboxymethylcellulose hydrogels. Cell Tissue Res 358: 527-539.

Gupta MS, Nicoll SB (2015) Duration of TGF- $\beta 3$ exposure impacts the chondrogenic maturation of human MSCs in photocrosslinked carboxymethylcellulose hydrogels. Ann Biomed Eng 43: 1145-1157.

Gupta MS, Nicoll SB (2013) Macromer concentration influences nucleus pulposus-like ECM elaboration and TGFBeta-3 family gene expression of hMSCs encapsulated in of photocrosslinked carboxymethylcellulose hydrogels. Trans Annu Meet Orthop Res Soc 38: 321.

Gupta MS, Cooper ES, Nicoll SB (2011) TGF-beta 3 stimulates cartilage matrix elaboration by human marrow-derived stromal cells encapsulated in photocrosslinked carboxymethylcellulose hydrogels: potential. Tissue Eng Part A 17: 2903-2910.

Guterl CC, See EY, Blanquer SB, Pandit A, Ferguson SJ, Benneker LM, Grijpma DW, Sakai D, Eglin D, Alini M, Iatridis JC, Grad S (2013) Challenges and strategies in the repair of ruptured annulus fibrosus. Eur Cell Mater 25: 1-21.

Heinze T, Koschella A (2005) Carboxymethyl ethers of cellulose and starch - a review. Macromol Symp 223: 13-40.

Iatridis JC, Nicoll SB, Michalek AJ, Walter BA, Gupta MS (2013) Role of biomechanics in intervertebral disc degeneration and regenerative therapies: what needs repairing in the disc and what are promising biomaterials for its repair? Spine J 13: 243-262.

Ibim SM, Uhrich KE, Bronson R, El-Amin SF, Langer RS, Laurencin CT (1998) Poly(anhydrideco-imides): in vivo biocompatibility in a rat model. Biomaterials 19: 941-951.

Jacobs JJ, Andersson GBJ, Bell J-E, Weinstein SL, Dormans JP, Gnatz SM, Lane N, Puzas JE, St Clair EW, Yelin EH (2008) United States bone and joint decade: The burden of musculoskeletal diseases in the United States. Rosemont IL: American Academy of Orthopaedic Surgeons.

Johannessen W, Cloyd JM, O'Connell GD, Vresilovic EJ, Elliott DM (2006) Trans-endplate nucleotomy increases deformation and creep response in axial loading. Ann Biomed Eng 34: 687696.

Johannessen W, Vresilovic EJ, Wright AC, Elliott DM (2004) Intervertebral disc mechanics are restored following cyclic loading and unloaded recovery. Ann Biomed Eng 32: 70-76.

Joshi A, Fussell G, Thomas J, Hsuan A, Lowman A, Karduna A, Vresilovic E, Marcolongo M (2006) Functional compressive mechanics of a PVA/PVP nucleus pulposus replacement. Biomaterials 27: 176184.

Kim DY, Kwon DY, Lee BNB, Seo HW, Kwon JS, Lee BNB, Han DK, Kim JH, Min BH, Park K, Kim MS (2012) Injectable in situ-forming hydrogels for a suppression of drug burst from drug-loaded microcapsules. Soft Matter 8: 3638-3648.

Kim K, Park S, Kim Y (2009) Disc height and segmental motion as risk factors for recurrent lumbar disc herniation. Spine (Phila Pa 1976) 34: 2674-2678.

Laudier D, Schaffler MB, Flatow EL, Wang VM (2006) Novel procedure for high-fidelity tendon histology. J Orthop Res 25: 390-395.

Lewis G (2012) Nucleus pulposus replacement and regeneration/repair technologies: present status 
and future prospects. J Biomed Mater Res B Appl Biomater 100: 1702-1720.

Likhitpanichkul M, Dreischarf M, Illien-Junger S, Walter BA, Nukaga T, Long RG, Sakai D, Hecht AC, Iatridis JC (2014) Fibrin-genipin adhesive hydrogel for annulus fibrosus repair: performance evaluation with large animal organ culture, in situ biomechanics, and in vivo degradation tests. Eur Cell Mater 28: 25-38.

Lin HA, Gupta MS, Varma DM, Gilchrist ML, Nicoll SB (2016) Lower crosslinking density enhances functional nucleus pulposus-like matrix elaboration by human mesenchymal stem cells in carboxymethylcellulose hydrogels. J Biomater Res A 104: 165-177.

Malhotra NR, Han WM, Beckstein J, Cloyd J, Chen W, Elliott DM (2012) An injectable nucleus pulposus implant restores compressive range of motion in the ovine disc. Spine (Phila Pa 1976) 37: E1099-1105. doi: 10.1097/BRS.0b013e31825cdfb7.

McGirt MJ, Eustacchio S, Varga P, Vilendecic M, Trummer M, Gorensek M, Ledic D, Carragee EJ (2009) A prospective cohort study of close interval computed tomography and magnetic resonance imaging after primary lumbar discectomy: factors associated with recurrent disc herniation and disc height loss. Spine (Phila Pa 1976) 34: 2044-2051.

Mironi-Harpaz I, Wang DY, Venkatraman S, Seliktar D (2012) Photopolymerization of cellencapsulating hydrogels: crosslinking efficiency versus cytotoxicity. Acta Biomater 8: 1838-1848.

Miyamoto T, Takahashi S, Ito $\mathrm{H}$, Inagaki $\mathrm{H}$, Noishiki Y (1989) Tissue biocompatibility of cellulose and its derivatives. J Biomed Mater Res 23: 125-133.

Panjabi M (1992) The stabilizing system of the spine. Part II. Neutral zone and instability hypothesis. J Spinal Disord 5: 390-397.

Panjabi MM, White AA (1980) Basic biomechanics of the spine. Neurosurgery 7: 76-93.

Parker SL, Grahovac G, Vukas D, Vilendecic M, Ledic D, McGirt, Matthew Carragee E (2016) Effect of an annular closure device (Barricaid) on same-level recurrent disk herniation and disk height loss after primary lumbar discectomy: two-year results of a multicenter prospective cohort study. Clin Spine Surg 29: 454-460.

Pérez-San Vicente A, Peroglio M, Ernst M, Casuso P, Loinaz I, Grande HJ, Alini M, Eglin D, Dupin D (2017) Self-healing dynamic hydrogel as injectable shock-absorbing artificial nucleus pulposus. Biomacromolecules 18: 2360-2370.

Reza AT, Nicoll SB (2010) Characterization of novel photocrosslinked carboxymethylcellulose hydrogels for encapsulation of nucleus pulposus cells. Acta Biomater 6: 179-186.

Schneider, C.A., Rasband, W.S., Eliceiri KW (2012) NIH Image to ImageJ: 25 years of image analysis. Nat Methods 9: 671-675.

Shin B-J (2014) Risk factors for recurrent lumbar disc herniations. Asian Spine J 8: 211-215.

Shin H, Olsen BD, Khademhosseini A (2012) The mechanical properties and cytotoxicity of cell-laden double-network hydrogels based on photocrosslinkable gelatin and gellan gum biomacromolecules. Biomaterials 33: 3143-3152.

Showalter BL, Malhotra NR, Vresilovic EJ, Elliott DM (2014) Nucleotomy reduces the effects of cyclic compressive loading with unloaded recovery on human intervertebral discs. J Biomech 47: 2633-2640.

Showalter BL, Elliott DM, Chen W, Malhotra NR (2015) Evaluation of an in situ gelable and injectable hydrogel treatment to preserve human disc mechanical function undergoing physiologic cyclic loading followed by hydrated recovery. J Biomech Eng 137: 081008. doi: 10.1115/1.4030530.

Siggelkow W, Faridi A, Spiritus K, Klinge U, Rath W, Klosterhalfen B (2003) Histological analysis of silicone breast implant capsules and correlation with capsular contracture. Biomaterials 24: 1101-1109.

Smith LJ, Gorth DJ, Showalter BL, Chiaro JA, Beattie EE, Elliott DM, Mauck RL, Chen W, Malhotra NR (2014) In vitro characterization of a stem-cellseeded triple-interpenetrating-network hydrogel for functional regeneration of the nucleus pulposus. Tissue Eng Part A 20: 1841-1849.

Stalling SS, Akintoye SO, Nicoll SB (2009) Development of photocrosslinked methylcellulose hydrogels for soft tissue reconstruction. Acta Biomater 5: 1911-1918.

Thomas JD, Fussell G, Sarkar S, Lowman AM, Marcolongo M (2010) Synthesis and recovery characteristics of branched and grafted PNIPAAmPEG hydrogels for the development of an injectable load-bearing nucleus pulposus replacement. Acta Biomater 6: 1319-1328.

Umehara S, Tadano S, Abumi K, Katagiri K, Kaneda K, Ukai T (1996) Effects of degeneration on the elastic modulus distribution in the lumbar intervertebral disc. Spine (Phila Pa 1976) 21: 811-819.

Varma DM (2016) Development of a redoxcrosslinked cellulose-based hydrogel for nucleus pulposus replacement and repair. The City College of New York, ProQuest Dissertations Publishing, 2016. 10159944.

Varma DM, Gold GT, Taub PJ, Nicoll SB (2014) Injectable carboxymethylcellulose hydrogels for soft tissue filler applications. Acta Biomater 10: 4996-5004.

Vogler EA (2012) Protein adsorption in three dimensions. Biomaterials 33: 1201-1237.

Wallace DG, Rosenblatt J, Ksander GA (1992) Tissue compatibility of collagen-silicone composites in a rat subcutaneous model. J Biomed Mater Res 26: 1517-1534.

Weber H (1994) The natural history of disc herniation and the influence of intervention. Spine (Phila Pa 1976) 19: 2234-2238.

Wilke HJ, Neef P, Caimi M, Hoogland T, Claes LE (1999) New in vivo measurements of pressures in the intervertebral disc in daily life. Spine (Phila Pa 1976) 24: 755-762.

Winter HH, Chambon F (1986) Analysis of linear viscoelasticity of a crosslinking polymer 
at the gel point. J Rheol (N Y N Y) 30: 367. doi. org/10.1122/1.549853.

Wortsman X, Wortsman J, Orlandi C, Cardenas G, Sazunic I, Jemec GBE (2012) Ultrasound detection and identification of cosmetic fillers in the skin. J Eur Acad Dermatol Venereol 26: 292-301.

\section{Discussion with Reviewers}

Nadeen Chahine: The biomechanical analysis evaluated 6 metrics of biomechanical evaluation of motion segments with and without hydrogel repair. Some measures (e.g. ROM, NZ stiffness and length, slow ramp stiffness) showed more sensitivity to injury and repair than others (e.g. compressive and tensile stiffness). Could you contextualize the importance of these different biomechanical measures? How do you prioritize these measures in developing and refining your biomaterial design for NP repair?

Authors: The biomaterial and structural integrity of the NP, AF and endplate all interact in defining the nonlinear structural biomechanical behaviors of the motion segment, which are quantified with the six parameters presented in this study. Nevertheless, some general assumptions can be made to interpret these findings. The ROM, NZ stiffness and length and slow ramp stiffness primarily reflect the biomechanical integrity of the NP, while the compressive and tensile stiffness might be influenced by the state of the NP and the AF, as suggested by the full restoration of compressive stiffness and partial restoration of the tensile stiffness with an AF repair strategy, where the NP was kept intact (Likhitpanichkul et al., 2014, Iatridis et al., 2013). The ROM and NZ length are indicative of overall filling of the NP and integrity of the disc, while the NZ stiffness also allows a comparison of the material properties of a replacement material to that of the native NP. For instance, the ROM and NZ length may be restored to native values with most NP replacements, yet a very stiff NP replacement device would also greatly influence NZ stiffness. A biomechanical mismatch between biomaterials and the native disc would result in stress concentrations that could cause mechanical damage to the structures surrounding the NP, especially the end plate and vertebral bodies. Thus, it is important to match as many biomechanical properties to the healthy condition as possible when developing a biomaterial for disc repair. Additionally, the disc height is also a clinically important parameter to determine the integrity and extent of degeneration and is associated with pain and disability.

David Eglin: The authors included, appropriately, the limitation of the bovine motion segments model in replicating the possible mechanical changes due to discectomy. It would be interesting to know the opinion of the authors on what could be a more representative ex vivo model.

Authors: The current model isolates the mechanical injury of the IVD to the NP to identify the effect of the hydrogel implant on restoring biomechanical properties of the NP with a relatively intact AF. Thus, currently the evaluation of this DNP hydrogel implant is limited to early to moderate stages of intervertebral disc disease. A more clinically representative model could include mechanical changes to the endplate and the $\mathrm{AF}$, as all three components of the disc undergo mechanical and biochemical changes from early to late stages of intervertebral disc degeneration.

David Eglin: It would be interesting to have the authors elaborate briefly on how replacement material may limit disc disease progression. Can we assume that resuming an "optimal" biomechanical environment of an IVD would be sufficient for stopping disease progression?

Authors: The stage of the disease and patient selection play an important role in determining the success of a treatment for disc degeneration. An acellular NP replacement material, such as the DNP described in this study, is most amenable to restoring the disc height and healthy biomechanical behaviors of the motion segment, and is most applicable to treating discs in the early to moderate stages of intervertebral disc degeneration, where the integrity of the $\mathrm{AF}$ and the endplate are maintained. The DNP is also amenable to bioactive formulations, including cell delivery, which are under development. A biological repair of the disc with a cell-laden NP replacement material could be more beneficial in limiting disease progression (Hudson et al., 2013, Iatridis et al., 2013). For later stages of the disease, an NP replacement combined with an AF repair might be necessary to limit disease progression and avoid a spinal fusion or a total disc replacement, which are likely required at late stages of degeneration.

\section{Additional References}

Hudson KD, Alimi M, Grunert P, Härtl R, Bonassar LJ (2013) Recent advances in biological therapies for disc degeneration: tissue engineering of the annulus fibrosus, nucleus pulposus and whole intervertebral discs. Curr Opin Biotechnol 24: 872-879.

Editor's note: The Scientific Editor responsible for this paper was Brian Johnstone. 